\title{
Half-graphs, other non-stable degree sequences, and the switch Markov chain
}

\author{
Péter L. Erdős ${ }^{*} \dagger \quad$ Ervin Győri* Tamás Róbert Mezei*,† \\ István Miklós*,t,ł, Dániel Soltész ${ }^{\dagger}, \S$ \\ Alfréd Rényi Institute of Mathematics, Eötvös Loránd Research Network (ELKH) \\ (a Hungarian Academy of Sciences Centre of Excellence) \\ Reáltanoda street 13-15, \\ H-1053 Budapest, Hungary \\ <erdos.peter,gyori.ervin,mezei.tamas.robert, \\ miklos.istvan, soltesz . daniel>@renyi.hu \\ ISZTAKI, Eötvös Loránd Research Network (ELKH) \\ Lágymányosi út 11, \\ H-1111 Budapest, Hungary
}

Submitted: Jun 15, 2020; Accepted: Jun 4, 2021; Published: Jul 2, 2021

(C) The authors. Released under the CC BY-ND license (International 4.0).

\begin{abstract}
One of the simplest methods of generating a random graph with a given degree sequence is provided by the Monte Carlo Markov Chain method using switches. The switch Markov chain converges to the uniform distribution, but generally the rate of convergence is not known. After a number of results concerning various degree sequences, rapid mixing was established for so-called $P$-stable degree sequences (including that of directed graphs), which covers every previously known rapidly mixing region of degree sequences.

In this paper we give a non-trivial family of degree sequences that are not $P$-stable and the switch Markov chain is still rapidly mixing on them. This family has an intimate connection to Tyshkevich-decompositions and strong stability as well.
\end{abstract}

Mathematics Subject Classifications: 05C30, 60J10, 68R10

\footnotetext{
*These authors were supported in part by the National Research, Development and Innovation Office, NKFIH grants K-116769, K-132696, and SNN-135643

$\dagger$ These authors were supported in part by the National Research, Development and Innovation Office, NKFIH grant KH-126853

${ }^{\ddagger}$ IM was supported in part by the National Research, Development and Innovation Office, NKFIH grant SNN-116095

$\S$ DS was supported in part by the National Research, Development and Innovation Office, NKFIH grants K-120706 and KH-130371.
} 


\section{Introduction}

An important problem in network science is to sample graphs with a given degree sequence (almost) uniformly. In this paper we study a Markov Chain Monte Carlo (MCMC) approach to this problem. The MCMC method can be successfully applied in many special cases. A vague description of this approach is that we start from an arbitrary graph with a given degree sequence and sequentially apply small random modifications that preserve the degree sequence of the graph. This can be viewed as a random walk on the space of realizations (graphs) of the given degree sequence. It is well-known that after sufficiently many steps the distribution over the state space is close to the uniform distribution. The goal is to prove that the necessary number of steps to take (formally, the mixing time of the Markov chain) is at most a polynomial of the length of the degree sequence.

In this paper we study the so-called switch Markov chain (also known as the swap Markov chain). For clarity, we refer to the degree sequence of a simple graph as an unconstrained degree sequence. For consistency, a graph with an unconstrained degree sequence is called an unconstrained graph, accordingly.

Throughout the paper, we work with finite graphs on labelled vertex sets. We will denote graphs with upper case letters (e.g. $G$ ), degree sequences (which are non-negative integer vectors) with bold-italic lower case letters (e.g. $\boldsymbol{d}$ ). Classes of graphs and classes of degree sequences are both denoted by upper case calligraphic letters (e.g. $\mathcal{H}$ ). We say that a graph $G$ is a realization of a degree sequence $\boldsymbol{d}$, if the degree sequence of $G$ is $\boldsymbol{d}$. For a degree sequence $\boldsymbol{d}$, we denote the set of all realizations of $\boldsymbol{d}$ by $\mathcal{G}(\boldsymbol{d})$. The $\ell^{1}$-norm of a vector $x$ is denoted by $\|x\|_{1}$.

For two graphs $G_{1}, G_{2}$ on the same labelled vertex set, we define their symmetric difference $G_{1} \triangle G_{2}$ with $V\left(G_{1} \triangle G_{2}\right)=V\left(G_{1}\right)=V\left(G_{2}\right)$ and $E\left(G_{1} \triangle G_{2}\right)=E\left(G_{1}\right) \triangle E\left(G_{2}\right)$.

Definition 1.1 (switch). For a bipartite or an unconstrained degree sequence $\boldsymbol{d}$, we say that two realizations $G_{1}, G_{2} \in \mathcal{G}(\boldsymbol{d})$ are connected by a switch, if

$$
\left|E\left(G_{1} \triangle G_{2}\right)\right|=4 .
$$

For directed graphs, beyond the classical switch operation, we also allow reversing an oriented 3-cycle (this is known as triple switch [5]).

A switch can be seen in Figure 1; for the precise definition of the switch Markov chain, see Definition 3.1. Clearly, if $G_{1}$ and $G_{2}$ are two graphs connected by a switch, then $F=E\left(G_{1}\right) \triangle E\left(G_{2}\right)$ is a cycle of length four (a $\left.C_{4}\right)$, and $E\left(G_{2}\right)=E\left(G_{1}\right) \Delta F$. Hence, the term switch is also used to refer to the operation of taking the symmetric difference with a given $C_{4}$. It should be noted, though, that only a minority of $C_{4}$ 's define a (valid) switch. The majority of $C_{4}$ 's do not preserve the degree sequence (if the $C_{4}$ does not alternate between edges of $G_{1}$ and $G_{2}$ ), or they introduce an edge which violates the constraints of the model (say, an edge inside one of the color classes in the bipartite case).

The question whether the mixing time of the switch Markov chain is short enough is interesting from both a practical and a theoretical point of view (although short enough 


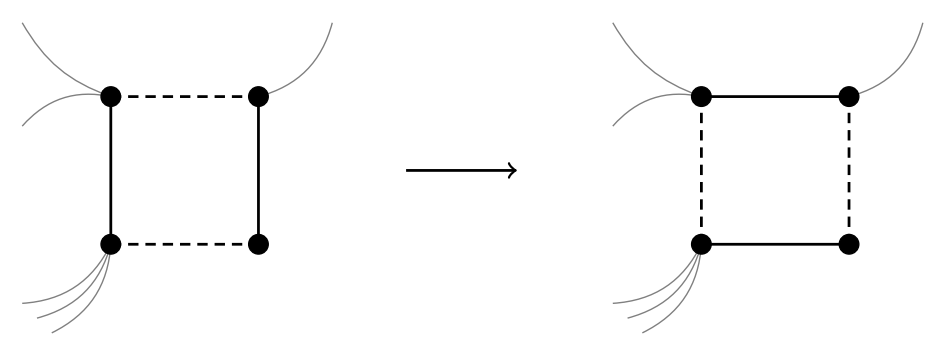

Figure 1: A switch (dashed lines emphasize missing edges)

depends greatly on the context). The switch Markov chain is already used in applications, hence rigorous upper bounds on its mixing time are much needed, even for special cases.

The switch Markov chain uses transitions which correspond to minimal perturbations. There are many other instances where the Markov chain of the smallest perturbations have polynomial mixing time, see [21]. However, it is unknown whether the mixing time of the switch Markov chain is uniformly bounded by a polynomial for every (unconstrained) degree sequence. Hence from a theoretical point of view, even an upper bound of $\mathcal{O}\left(n^{10}\right)$ on the mixing time of the switch Markov chain would be considered a great success, even though in practice it is only slightly better than no upper bound at all.

The present paper is written from a theoretical point of view and should be considered as a step towards answering the following question.

Question 1.2 (Kannan, Tetali, and Vempala [17]). Is the switch Markov chain rapidly mixing on the realizations of all graphic degree sequences?

Jerrum and Sinclair introduced the notion of $P$-stability in their seminal paper [16], and they proved that the Jerrum-Sinclair chain is rapidly mixing on such degree sequences. Jerrum, Sinclair, and McKay [15] recognized there exists a non- $P$-stable degree sequence which has a unique realization (trivially rapidly mixing): take

$$
(2 n-1,2 n-2, \ldots, n+1, n, n, n-1, \ldots, 2,1) \in \mathbb{N}^{2 n} .
$$

In its unique realization, the first $n$ vertices form a clique, while the remaining vertices form an independent set.

Definition 1.3 $(\boldsymbol{P}$-stability). Let $\mathcal{D}$ be an infinite set of unconstrained/bipartite/directed degree sequences. We say that $\mathcal{D}$ is $\boldsymbol{P}$-stable, if there exists a polynomial $p$ over the real numbers such that for any $n \in \mathbb{N}$ and any degree sequence $\boldsymbol{d} \in \mathcal{D}$ on $n$ vertices we have

$$
\left|\mathcal{G}(\boldsymbol{d}) \cup\left(\bigcup_{x, y \in[n], x \neq y} \mathcal{G}\left(\boldsymbol{d}-\mathbb{1}_{x}-\mathbb{1}_{y}\right)\right)\right| \leqslant p(n) \cdot|\mathcal{G}(\boldsymbol{d})|,
$$

where $\mathbb{1}_{x}$ is the $x^{\text {th }}$ unit vector.

For bipartite graphs, we get an equivalent definition if we replace the inequality with $\left|\mathcal{G}(\boldsymbol{d}) \cup\left(\bigcup_{x, y \in[n], x \neq y} \mathcal{G}\left(\boldsymbol{d}+\mathbb{1}_{x}+\mathbb{1}_{y}\right)\right)\right| \leqslant p^{\prime}(n) \cdot|\mathcal{G}(\boldsymbol{d})|\left(\right.$ where $p^{\prime}$ only depends on $\mathcal{D}$ ) or 
with $\left|\mathcal{G}(\boldsymbol{d}) \cup\left(\bigcup_{x, y \in[n], x \neq y} \mathcal{G}\left(\boldsymbol{d}+\mathbb{1}_{x}-\mathbb{1}_{y}\right)\right)\right| \leqslant p^{\prime \prime}(n) \cdot|\mathcal{G}(\boldsymbol{d})|$ (again, $p^{\prime \prime}$ only depends on $\mathcal{D}$ ): the sets whose sizes are estimated in the inequalites can be mapped to one another by adding/removing one or two edges; since two edges can only be chosen in $n^{4}$ ways, we only overcounted by at most a polynomial factor, which means that the cardinalities on the left hand sides are at most a polynomial factor apart.

There is a long line of results where the rapid mixing of the switch Markov chain is proven for certain degree sequences, see $[2,19,13,6,7,12]$. Some of these results were unified, first by Amanatidis and Kleer [1], who established rapid mixing for so-called strongly stable classes of degree sequences of unconstrained and bipartite graphs (definition given in Section 7).

The most general result at the time of writing is proved by Erdős, Greenhill, Mezei, Miklós, Soltész, and Soukup:

Theorem 1.4 ([4]). The switch Markov chain is rapidly mixing on sets of unconstrained, bipartite, and directed degree sequences that are $\boldsymbol{P}$-stable (see Definition 8.3).

For the sake of being less redundant, the phrase "D is rapidly mixing" shall carry the same meaning as "switch Markov chain is rapidly mixing on $\mathcal{D}$ ".

Our goal in this paper is to start extending the set of rapidly mixing bipartite degree sequences beyond $P$-stability. The degree sequence $(1)$ can naturally be turned into a bipartite one by assigning the role of the two color classes to the clique and the independent set, and then removing the edges of the clique.

Definition 1.5. Let us define a bipartite degree sequence:

$$
\begin{aligned}
\boldsymbol{h}_{0}(n) & :=\left(\begin{array}{ccccccc}
1 & 2 & 3 & \cdots & n-2 & n-1 & n \\
n & n-1 & n-2 & \cdots & 3 & 2 & 1
\end{array}\right) \\
\mathcal{H}_{0} & :=\left\{\boldsymbol{h}_{0}(n) \mid n \in \mathbb{N}\right\}
\end{aligned}
$$

Let $A_{n}=\left\{a_{1}, \ldots, a_{n}\right\}$ and $B_{n}=\left\{b_{1}, \ldots, b_{n}\right\}$, often denoted simply $A$ and $B$. We label the vertices of $\boldsymbol{h}_{0}(n)$ such that $A$ is the first and $B$ is the second color class, with $\operatorname{deg}_{\boldsymbol{h}_{0}(n)}\left(a_{i}\right)=n+1-i$ and $\operatorname{deg}_{\boldsymbol{h}_{0}(n)}\left(b_{i}\right)=i$ for $i \in[1, n]$. The unique realization $H_{0}(n)$, also known as the half-graph, is displayed on Figure 2.
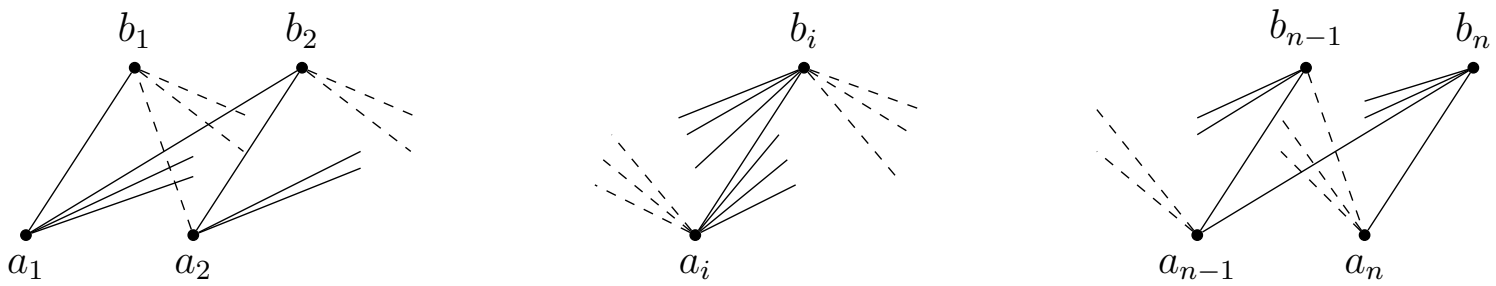

Figure 2: The unique realization $H_{0}(n)$ of $\boldsymbol{h}_{0}(n)$ is isomorphic to the half-graph. Dashed line segments represent non-edges.

In this paper, we conduct a detailed study of $\boldsymbol{h}_{0}(n)$ and its neighborhood. Before presenting our main results, let us get familiar with two interesting properties of $\boldsymbol{h}_{0}(n)$. 


\subsection{Simple examples for rapidly mixing non-stable bipartite classes}

Let $\mathbb{1}_{x}$ be the vector which takes 1 on $x$ and zero everywhere else. In Corollary 6.2 we solve a linear recursion, which shows that

$$
\left|\mathcal{G}\left(\boldsymbol{h}_{0}(n)-\mathbb{1}_{a_{1}}-\mathbb{1}_{b_{n}}\right)\right|=\Theta\left(\left(\frac{1+\sqrt{5}}{2}\right)^{n}\right),
$$

therefore $\mathcal{H}_{0}$ is not $P$-stable.

Although $\boldsymbol{h}_{0}(n)$ seems very pathological as an example for a non-stable degree sequence, it is a source of more interesting examples. It is well-known that the random walk on the vertices of a hypercube $\{0,1\}^{n}$ is rapidly mixing. As pointed out to us by an anonymous reviewer, this process can be modelled with the switch Markov chain. Take $H_{0}(n)$ and replace $a_{i} b_{i}$ by a pair of independent edges simultaneously for all $i$ : the degree sequence of the obtained graph is

$$
\boldsymbol{g}(n):=\left(\begin{array}{ccccccc}
1 & 1 & 3 & 3 & \cdots & 2 n-1 & 2 n-1 \\
2 n-1 & 2 n-1 & 2 n-3 & 2 n-3 & \cdots & 1 & 1
\end{array}\right) .
$$

To each vertex of the $n$-dimensional hypercube, we can assign a realization of $g(n)$ as follows: replace $a_{i} b_{i}$ with two parallel or two crossing edges depending on whether the $i^{\text {th }}$ coordinate of the vertex of the hypercube is 0 or 1 . The transition of the hypercube in the $i^{\text {th }}$ coordinate corresponds to the switch on the two edges replacing $a_{i} b_{i}$. Because the random-walk on a hypercube is rapidly mixing, the switch Markov chain is rapidly mixing on $\{\boldsymbol{g}(n) \mid n \in \mathbb{N}\}$. Moreover, by solving yet another a linear recursion, one can verify that $\{\boldsymbol{g}(n) \mid n \in \mathbb{N}\}$ is not $P$-stable.

In Section 2.2, we will draw the curtain on the explanation behind the behavior of $\boldsymbol{h}_{0}(n)$ and $\boldsymbol{g}(n)$. In the meantime, we present the main results of the paper.

\subsection{Results}

If $\boldsymbol{d}$ is the degree sequence of the bipartite graph $G[A, B]$, then $\boldsymbol{d}=\left(\boldsymbol{d}^{A} ; \boldsymbol{d}^{B}\right)$ is split across the bipartition as well, and it is called a splitted bipartite degree sequence [8]. The disjoint vertex classes $A$ and $B$ are not interchangeable, their order is fixed in the splitted bipartite graph $G[A, B]$. We say that $G[A, B]$ is the empty bipartite graph if both $A=B=\varnothing$.

Definition 1.6. For a set $\mathcal{D}$ of bipartite degree sequences and any $k \in \mathbb{N}$, let

$$
\begin{aligned}
& \mathbb{B}_{2 k}(\mathcal{D})=\bigcup_{\boldsymbol{d} \in \mathcal{D}}\left\{\boldsymbol{e}: \operatorname{Dom}(\boldsymbol{d}) \rightarrow \mathbb{N} \mid\|d-e\|_{1} \leqslant 2 k,\left\|e^{A}\right\|_{1}=\left\|e^{B}\right\|_{1}\right\} \\
& \mathbb{S}_{2 k}(\mathcal{D})=\bigcup_{\boldsymbol{d} \in \mathcal{D}}\left\{\boldsymbol{e}: \operatorname{Dom}(\boldsymbol{d}) \rightarrow \mathbb{N} \mid\|d-e\|_{1}=2 k,\left\|e^{A}\right\|_{1}=\left\|e^{B}\right\|_{1}\right\}
\end{aligned}
$$

be the (closed) ball and sphere of radius $2 k$ around $\mathcal{D}$. The requirement that $\left\|e^{A}\right\|_{1}=\left\|e^{B}\right\|_{1}$, i.e., that the sum of the degrees on the two sides be equal is necessary for graphicality. 
We will show in Section 5 that neighborhoods of $\mathcal{H}_{0}=\left\{\boldsymbol{h}_{0}(n) \mid n \in \mathbb{N}\right\}$ are rapidly mixing:

Theorem 1.7. For any fixed $k$, the switch Markov chain is rapidly mixing on the bipartite degree sequences in $\mathbb{B}_{2 k}\left(\mathcal{H}_{0}\right)$.

Next, we show that even though balls of constant size around $\mathcal{H}_{0}$ are rapidly mixing, $\mathbb{S}_{2 k}\left(\mathcal{H}_{0}\right)$ contains a degree sequence which is not $P$-stable.

Definition 1.8. For all $k, n \in \mathbb{N}$ where $k<n$ let

$$
\begin{aligned}
\boldsymbol{h}_{k}(n) & :=\boldsymbol{h}_{0}(n)-k \cdot \mathbb{1}_{a_{1}}-k \cdot \mathbb{1}_{b_{n}} \\
\mathcal{H}_{k} & :=\left\{\boldsymbol{h}_{k}(n) \mid k \leqslant n \in \mathbb{N}^{+}\right\}
\end{aligned}
$$

be a bipartite degree sequence and a class of bipartite degree sequences, respectively.

Theorem 1.9. The class of degree sequences $\mathcal{H}_{k}$ is not $P$-stable for any $k \in \mathbb{N}$.

\subsection{Outline}

The rest of the paper is organized as follows.

- As promised at the end of Section 1.1, we introduce the Tyshkevich-decomposition of bipartite graphs in Section 2.

- In Section 3 we introduce the switch Markov chains, some related definitions, and Sinclair's result on mixing time.

- Section 4 describes the structure of realizations of degree sequences from $\mathbb{B}_{2 k}\left(\boldsymbol{h}_{0}\right)$, which is then used by Sections 5 and 6 to prove Theorems 1.7 and 1.9, respectively.

- In Section 7 we provide further motivation for studying $\boldsymbol{h}_{0}(n)$ and alternating cycles covers. We show that every graph which is not stable in a certain sense contains a copy of $H_{0}(\ell)$. The goal of this section is to inspire further research of the switch Markov chain (on bipartite graphs).

- Section 8 describes how $\boldsymbol{h}_{0}(n)$ relates to previous research. Possible generalizations of Theorem 1.7 are conjectured.

\section{Properties of Tyshkevich-decompositions}

\subsection{Tyshkevich-decomposition of bipartite graphs}

Let $G$ be a unconstrained graph. It is a split graph if there is a partition $V(G)=A \uplus B$ $(A \neq \varnothing$ or $B \neq \varnothing)$ such that $A$ is a clique and $B$ is an independent set in $G$. Split graphs were first studied by Földes and Hammer [10], who determined that being split is a property of the degree sequence $\boldsymbol{d}$ of $G$. Note, that the partition is not necessarily 
unique, but the size of $A$ is determined up to a +1 additive constant, see [14]. A split graph endowed with an ordered bipartition is called a splitted graph, denoted by $(G, A, B)$. In addition to [10], Tyshkevich ${ }^{1}$ and Chernyak [23] also determined that the property of being a split graph is a property of the degree sequence, thus every realization of a split degree sequence is a split graph.

Tyshkevich and co-authors have extensively studied a composition operator denoted by "o" on (split) graphs; these results are nicely collected in [22]. The composition $(G, A, B) \circ H$ takes the disjoint union of a split graph and an unconstrained graph, and joins every vertex in $A$ to every vertex of $H$. It is easy to see that the composition of two split graphs is also a split graph. A fundamental result on this operator is that any unconstrained graph can be uniquely decomposed into the non-commutative composition of split graphs and possibly an indecomposable unconstrained graph as the last factor.

Let us slightly change the conventional notation $G[A, B]$ to also signal that the color classes $A$ and $B$ are ordered (2-colored); to emphasize this, we may refer to such graphs as splitted bipartite graphs. Observe, that a function $\Psi$ removing the edges of the clique on $A$ from $(G, A, B)$ produces a splitted bipartite graph $G[A, B]$. Erdős, Miklós, and Toroczkai [9] adapted the results about split graphs and the composition operator $\circ$ to splitted bipartite graphs via the bijection given by $\Psi$.

Definition 2.1. Given two splitted bipartite graphs $G[A, B]$ and $H[C, D]$ with disjoint vertex sets, we define their (Tyshkevich-) composition $G[A, B] \circ H[C, D]$ as the bipartite graph

$$
G[A, B] \circ H[C, D]:=G[A, B] \cup H[C, D]+\{a d \mid a \in A, d \in D\} .
$$

The o operator is clearly associative, but not commutative. We say that a bipartite graph is indecomposable if it cannot be written as a composition of two non-empty bipartite graphs.

Lemma 2.2 ([9], adapted from Theorem 2(i) in [22]). Let $G[A, B]$ be a bipartite graph with degree sequence $d=\left(d^{A}, d^{B}\right)$, where both $d^{A}$ and $d^{B}$ are in non-increasing order. Then $G[A, B]$ is decomposable if and only if there exists $p, q \in \mathbb{N}$ such that $0<p+q<|A|+|B|$, $0 \leqslant p \leqslant|A|, 0 \leqslant q \leqslant|B|$, and

$$
\sum_{i=1}^{p} d_{i}^{A}=p(|B|-q)+\sum_{|B|-q+1}^{|B|} d_{i}^{B} .
$$

Theorem 2.3 ([9], adapted from Corollaries 6 and 9 in [22]).

(i) Any splitted bipartite degree sequence d can be uniquely decomposed in the form

$$
d=\alpha_{1} \circ \cdots \circ \alpha_{k}
$$

where $\alpha_{i}$ is an indecomposable splitted bipartite degree sequence for $i=1, \ldots, k$.

\footnotetext{
${ }^{1}$ During the writing of this paper, we were greatly saddened to learn that Professor Tyshkevich passed away in November, 2019.
} 
(ii) Any realization $G$ of $d$ can be represented in the form

$$
G=G\left[A_{1}, B_{1}\right] \circ \cdots \circ G\left[A_{k}, B_{k}\right]
$$

where $G\left[A_{i}, B_{i}\right]$ is a realization of $\alpha_{i}$.

(iii) Any valid bipartite switch of $G$ is a valid bipartite switch of $G\left[A_{i}, B_{i}\right]$ for some $i$.

It follows from the previous theorem that indecomposability is determined by the degree sequence. Lemma 2.2 gives an explicit characterization of such splitted bipartite degree sequences.

Definition 2.4. Let $\overline{\mathcal{D}^{\circ}}$ be the finite closure of $\mathcal{D}$ under the composition operator $\circ$.

The following theorem is a due to Erdős, Miklós, and Toroczkai.

Theorem 2.5 (Theorem 3.6 in [9]). If $\mathcal{D}$ is rapidly mixing, then so is $\overline{\mathcal{D}^{\circ}}$.

Theorem 2.5 is a simple consequence of [8, Theorem 5.1]. By Theorem 2.5, for a class of degree sequences $\mathcal{D}$ to be rapidly mixing it is sufficient that indecomp $(\mathcal{D})$ is rapidly mixing, where

$$
\operatorname{indecomp}(\mathcal{D}):=\{\alpha \mid \alpha \text { is an indecomposable component of some } d \in \mathcal{D}\} .
$$

Because the number of realizations is independent of the internal order of the bipartition, we revert to using "bipartite degree sequence" instead of the cumbersome "splitted bipartite degree sequence". From now on, bipartite graphs and their degree sequences are assumed to be splitted.

\subsection{Non-stability of Tyshkevich-compositions}

As promised, we now revisit the two examples in Section 1.1. The complete graph on two vertices $K_{2}$ is naturally a split graph. Observe, that

$$
\boldsymbol{h}_{0}(n)=\overbrace{(1 ; 1) \circ \ldots \circ(1 ; 1)}^{n} \text {. }
$$

Recall from Definition 1.5, that the unique realization of $\boldsymbol{h}_{0}(n)$ is

$$
H_{0}(n)=\overbrace{K_{2} \circ \ldots \circ K_{2}}^{n}
$$

Note, that $(1 ; 1)=(0 ; \varnothing) \circ(\varnothing ; 0)$, so the indecomposable decomposition of $\boldsymbol{h}_{0}(n)$ has $2 n$ components. Theorem 2.3 implies that $H_{0}(n)$ is the only realization of $\boldsymbol{h}_{0}(n)$. This innocent looking example leads to the following result:

Lemma 2.6. For any class $\mathcal{D}$ of bipartite degree sequences, $\overline{\mathcal{D}^{\circ}}$ is not P-stable (except if $\alpha^{A}=\varnothing$ for all $\alpha \in \mathcal{D}$ or $\beta^{B}=\varnothing$ for all $\left.\beta \in \mathcal{D}\right)$. 
Proof. Take $\alpha, \beta \in \mathcal{D}$ such that $\alpha^{A} \neq \varnothing$ and $\beta^{B} \neq \varnothing$. Let

$$
\boldsymbol{d}(r)=\overbrace{(\alpha \circ \beta) \circ \ldots \circ(\alpha \circ \beta)}^{r} .
$$

From Theorem 2.3 it follows that

$$
|\mathcal{G}(\boldsymbol{d}(r))|=|\mathcal{G}(\boldsymbol{\alpha})|^{r} \cdot|\mathcal{G}(\boldsymbol{\beta})|^{r} .
$$

Let $G=\left(G_{1} \circ G_{2}\right) \circ \ldots \circ\left(G_{2 r-1} \circ G_{2 r}\right)$ be an arbitrary realization of $\boldsymbol{d}(r)$ where $G_{2 i-1} \in \mathcal{G}(\alpha)$ and $G_{2 i} \in \mathcal{G}(\beta)$. Recall that $\boldsymbol{h}_{0}(r)-\mathbb{1}_{a_{1}}-\mathbb{1}_{b_{r}}$ has exponentially many realizations, see Equation (2).

Let $a_{i}$ be the first vertex of the first class of $G_{2 i-1}$ and let $b_{i}$ be the first vertex of the second class of $G_{2 i}$ (for $i \in[1, r]$ ). By the definition of the Tyshkevich-composition, these choices are the same for any two realizations of $\boldsymbol{d}(r)$.

Observe, that $G\left[\left\{a_{1}, \ldots, a_{r}\right\},\left\{b_{1}, \ldots, b_{r}\right\}\right]$ is an induced copy of $H_{0}(r)$. By replacing this subgraph with a realization of $\boldsymbol{h}_{0}(r)-\mathbb{1}_{a_{1}}-\mathbb{1}_{b_{r}}$, an exponential number of realizations of $\boldsymbol{d}(r)-\mathbb{1}_{a_{1}}-\mathbb{1}_{b_{r}}$ are obtained; however, because the substitution does not change the components $G_{2 i-1}$ and $G_{2 i}$ for any $i, G$ is recoverable from such realizations. In other words, every realization of some $\boldsymbol{d}^{\prime} \in \mathbb{S}_{2}(\boldsymbol{d}(r))$ is obtained from at most one realization of $\boldsymbol{d}(r)$, so $\mathcal{D}$ cannot be $P$-stable.

The degree sequence $\boldsymbol{g}(n)$ was obtained by replacing $a_{i} b_{i}$ with two independent edges (denoted as $2 K_{2}$ ). Therefore Lemma 2.6 applies to $\{\boldsymbol{g}(n) \mid n \in \mathbb{N}\}$ :

$$
\boldsymbol{g}(n)=\overbrace{(1,1 ; 1,1) \circ \ldots \circ(1,1 ; 1,1)}^{n}
$$

Naturally, $\overbrace{2 K_{2} \circ \ldots \circ 2 K_{2}}^{n}$ is a realization of $\boldsymbol{g}(n)$ and all $2^{n}$ realizations of $\boldsymbol{g}(n)$ are isomorphic to it (Theorem 2.3).

Theorem 1.9 is not, however, a simple consequence of Lemma 2.6:

Lemma 2.7. The bipartite degree sequence $\boldsymbol{h}_{k}(n)$ is indecomposable for $0<k<n$.

Proof. Via Lemma 2.2. Suppose $\boldsymbol{h}_{k}(n)$ is decomposable. Substituting into (3), we get

$$
\left(\begin{array}{c}
n+1 \\
2
\end{array}\right)-k-\left(\begin{array}{c}
n-p+1 \\
2
\end{array}\right)+\max \{k-p, 0\}=p(n-q)+\left(\begin{array}{c}
q+1 \\
2
\end{array}\right)-\max \{k-n+q, 0\}
$$

if and only if

$$
\max \{k-p, 0\}+\max \{k-n+q, 0\}-k=\left(\begin{array}{c}
q-p+1 \\
2
\end{array}\right) .
$$

A short case analysis shows that the right hand side is larger than the left hand side. 


\section{The switch Markov chain}

For the precise definition of Markov chains and an introduction to their theory, the reader is referred to Durrett [3]. To define the unconstrained and bipartite switch Markov chains, it is sufficient to define their transition matrices.

Definition 3.1 (unconstrained/bipartite switch Markov chain). Let $\boldsymbol{d}$ be an unconstrained or bipartite degree sequence on $n$ vertices. The state space of the switch Markov chain $\mathcal{M}(\boldsymbol{d})$ is $\mathcal{G}(\boldsymbol{d})$. The transition probability between two different states of the chain is nonzero if and only if the corresponding realizations are connected by a switch, and in this case this probability is $\frac{1}{6}\left(\begin{array}{c}n \\ 4\end{array}\right)^{-1}$. The probability that the chain stays at a given state is one minus the probability of leaving the given state.

An algorithmic description of the chain is as follows: choose 4 vertices uniformly and randomly, there are $\left(\begin{array}{l}n \\ 4\end{array}\right)$ possibilities. There are 3 ways to embed a $C_{4}$ into a $K_{4}$, choose one embedding randomly. With probability $\frac{1}{2}$, try to switch on the chosen $C_{4}$.

It is well-known that any two realizations of an unconstrained or bipartite degree sequence can be transformed into one-another through a series of switches. The space of realizations of a directed degree sequence is connected if triple switches are allowed (besides the usual directed switches).

The switch Markov chains defined are irreducible (connected), symmetric, reversible, and lazy. Their unique stationary distribution is the uniform distribution $\pi \equiv|\mathcal{G}(\boldsymbol{d})|^{-1}$.

Definition 3.2. The mixing time of a Markov chain $\mathcal{M}=(\Omega, P)$ on state space $\Omega$ and transition matrix $P$ with stationary distribution $\pi$ is

$$
\tau_{\mathcal{M}}(\varepsilon)=\min \left\{t_{0}: \forall x \forall t \geqslant t_{0} \quad\left\|P^{t}(x, \cdot)-\pi\right\|_{1} \leqslant \varepsilon\right\},
$$

where $P^{t}(x, y)$ is the probability that when $\mathcal{M}$ is started from $x$, then the chain is in $y$ after $t$ steps.

Definition 3.3. The switch Markov chain is said to be rapidly mixing on an infinite set of degree sequences $\mathcal{D}$ if there exists a fixed polynomial poly $\left(n, \log \varepsilon^{-1}\right)$ which bounds the mixing time of the switch Markov chain on $\mathcal{G}(\boldsymbol{d})$ for any $\boldsymbol{d} \in \mathcal{D}$ (where $n$ is the length of d).

Sinclair's seminal paper describes a combinatorial method to bound the mixing time.

Definition 3.4 (Markov graph). Let $G(\mathcal{M}(\boldsymbol{d}))$ be the graph whose vertices are realizations of $\boldsymbol{d}$ and two vertices are connected by an edge if the switch Markov chain on $\mathcal{G}(\boldsymbol{d})$ has a positive transition probability between the two realizations.

Let $\Gamma$ be a set of paths in $\mathcal{M}(\boldsymbol{d})$. We say that $\Gamma$ is a canonical path system if for any two realizations $G, H \in \mathcal{G}(\boldsymbol{d})$ there is a unique $\gamma_{G, H} \in \Gamma$ which joins $G$ to $H$ in the Markov graph. The load of $\Gamma$ is defined as

$$
\rho(\Gamma)=\max _{P(e) \neq 0} \frac{|\{\gamma \in \Gamma: e \in E(\gamma)\}|}{|\mathcal{G}(\boldsymbol{d})| \cdot P(e)},
$$


where $P(e)$ is the transition probability assigned to the edge $e$ of the Markov graph (this is well-defined because the studied Markov chains are symmetric). The next lemma follows from Proposition 1 and Corollary 4 of Sinclair [20].

Lemma 3.5. If $\Gamma$ is a canonical path system for $\mathcal{M}(\boldsymbol{d})$ then

$$
\tau_{\mathcal{M}(\boldsymbol{d})}(\varepsilon) \leqslant \rho(\Gamma) \cdot \ell(\Gamma) \cdot\left(\log (|\mathcal{G}(\boldsymbol{d})|)+\log \left(\varepsilon^{-1}\right)\right),
$$

where $\ell(\Gamma)$ is the length of the longest path in $\Gamma$.

Obviously, $\log (|\mathcal{G}(\boldsymbol{d})|) \leqslant n^{2}$, henceforth we focus on bounding $\rho(\Gamma)$ and $\ell(\Gamma)$ by a polynomial of $n$.

\section{Flow representation}

In this section we work with directed graphs, so let us fix the related notation first. Let $F$ be a directed graph. A directed edge $u v \in \vec{E}(F)$ points from $u$ to $v$. The in- and out-degrees of a vertex $v \in V(F)$ are denoted by $\varrho_{F}(v)$ and $\delta_{F}(v)$. For a subset of vertices $S \subseteq V(F)$, let $\varrho_{F}(S)$ be the number of edges $u v \in \vec{E}(F)$ such that $u \in V \backslash S$ and $v \in S$. Similarly, $\delta_{F}(S)$ is the number of edges $u v \in \vec{E}(F)$ such that $u \in S$ and $v \in V \backslash S$.

Theorem 4.1 (directed edge version of Menger's theorem [18]). Let $F$ be a directed graph (parallel and oppositely directed edges are allowed) with two distinct special vertices $s$ and $t$. There exists $k$ edge-disjoint directed paths from $s$ to $t$ if and only if for every $S \subset V(F)$ such that $s \in S$ and $t \notin S$ we have

$$
\delta_{F}(S) \geqslant k \text {. }
$$

Definition 4.2 (Integer 0-1 flows in directed graphs). Suppose $F$ is a directed graph. An integer 0-1 flow is a subgraph $H \subseteq F$. If $k=\sum_{v \in V(F)}\left(\delta_{H}(v)-\varrho_{H}(v)\right)^{+}$, then $H$ is called a $k$-flow. A vertex $s$ for which $\left(\delta_{H}(s)-\varrho_{H}(s)\right)^{+}>0$ is a source, a vertex $t$ for which $\left(\delta_{H}(t)-\varrho_{H}(t)\right)^{-}>0$ is a sink. A vertex $v$ conserves the flow if $\delta_{H}(t)=\varrho_{H}(t)$.

Lemma 4.3. The union of $k$ edge-disjoint paths of $F$ is a $k$-flow. If the underlying graph $F$ is acyclic, then a $k$-flow can always be decomposed into $k$ edge-disjoint paths.

Proof. The first statement is trivial. To decompose a $k$-flow $H$, we will use recursion. If $k>1$, then choose an arbitrary vertex $u$ for which $\delta_{H}(u)>\varrho_{H}(u)$. Let $S$ be the set of vertices which can be reached from $u$. Then we have $\delta_{F}(S)=0$, thus

$$
\sum_{v \in S} \delta_{H}(v) \leqslant \sum_{v \in S} \varrho_{H}(v)
$$

Since the out-degree of $u$ is larger than its in-degree, there exists a vertex $v$ for which $\delta_{H}(v)<\varrho_{H}(v)$. Let $P$ be the shortest path from $u$ to $v$, and remove the edges of $P$ from $H$, which decreases the size of the flow by 1 . If $H$ is a 0 -flow in $F$, but $E(H) \neq \varnothing$, then $H$ is Eulerian, so it contains a directed cycle, which is a contradiction. Therefore the outlined procedure finds a set of edge-disjoint paths which completely cover the flow. 
Theorem 4.4. Given a directed acyclic graph $F$, there exists a subgraph $H \subseteq F$ with prescribed in- and out-degree sequences $\varrho_{H}$ and $\delta_{H}$ if and only if for every $S \subseteq V(F)$

$$
\delta_{F}(S) \geqslant \sum_{v \in S}\left(\delta_{H}(v)-\varrho_{H}(v)\right)
$$

Proof. Follows from Menger's theorem (Theorem 4.1) and Lemma 4.3. Let

$$
f(v):=\delta_{H}(v)-\varrho_{H}(v)
$$

for every $v \in V(F)$. Add two auxiliary vertices $s$ and $t$ to $F$, and for every $v \in V(F)$ add $f(v)^{+}$copies of $s v$ and $f(v)^{-}$copies of $v t$ to the edge set; let $F^{\prime}$ denote the obtained graph. The desired $H$ exists if and only if there are $\sum_{v \in V(F)} f(v)^{+}$edge-disjoint paths from $s$ to $t$ in $F^{\prime}$. For any $S \subseteq V(F) \cup\{s\}$ such that $s \in S$, we must have:

$$
\begin{gathered}
\delta_{F^{\prime}}(S) \geqslant \sum_{v \in V(F)} f(v)^{+} \\
\delta_{F}(S-s)+\sum_{v \in V(F) \backslash S} f(v)^{+}+\sum_{v \in S-s} f(v)^{-} \geqslant \sum_{v \in S-s} f(v)^{+}+\sum_{v \in V(F) \backslash S} f(v)^{+} \\
\delta_{F}(S-s) \geqslant \sum_{v \in S-s} f(v)
\end{gathered}
$$

The last inequality implies (7).

It will be more convenient to work with $k$-flows then an arbitrary decomposition of the flow into $k$ edge-disjoint paths. Let us introduce a flow representation of realizations of bipartite degree sequences defined on $A_{n}$ and $B_{n}$ as their first and second color classes, respectively. Let us define the directed acyclic graph $F_{n}$, which is closely related to $H_{0}(n)$.

Definition 4.5. Let $F:=F_{n}=\left(A_{n}, B_{n}, \vec{E}\right)$ be a directed bipartite graph such that

- $a_{i} b_{j} \in \vec{E}(F)$ if and only if $i \leqslant j$,

- $b_{j} a_{i} \in \vec{E}(F)$ if and only if $j<i$.

The subgraph formed by the edges of $F_{n}$ leaving $A_{n}$ is an orientation of $H_{0}(n)$.

In general, for any subgraph $H \subseteq F=F_{n}$ and any subset of vertices $S \subseteq V(F)$, a simple double counting argument shows that

$$
\sum_{v \in S}\left(\delta_{H}(v)-\varrho_{H}(v)\right)=\delta_{H}(S)-\varrho_{H}(S)
$$

Definition 4.6. A flow realization of a splitted bipartite degree sequence $\boldsymbol{d}=\left(\boldsymbol{d}^{A_{n}} ; \boldsymbol{d}^{B_{n}}\right)$ is a flow $H$ in $F_{n}$ which satisfies

$$
\delta_{H}\left(a_{i}\right)-\varrho_{H}\left(a_{i}\right)=\operatorname{deg}_{H_{0}(n)}\left(a_{i}\right)-\operatorname{deg}_{\boldsymbol{d}}\left(a_{i}\right),
$$




$$
\delta_{H}\left(b_{i}\right)-\varrho_{H}\left(b_{i}\right)=\operatorname{deg}_{\boldsymbol{d}}\left(b_{i}\right)-\operatorname{deg}_{H_{0}(n)}\left(b_{i}\right),
$$

for every $a_{i} \in A_{n}$ and every $b_{i} \in B_{n}$, respectively. Recall from Definition 1.5, that $A_{i}=\left\{a_{1}, \ldots, a_{i}\right\}$ and $B_{i}=\left\{b_{1}, \ldots, b_{i}\right\}$. Let $\bar{A}_{i}:=A_{n} \backslash A_{i}$ and $\bar{B}_{i}:=B_{n} \backslash B_{i}$. Also, let $U_{i}:=A_{i} \cup B_{i}$ and $\bar{U}_{i}:=\bar{A}_{i} \cup \bar{B}_{i}$.

Observe, that $\varrho_{F}\left(U_{i}\right)=0$ and $\delta_{F}\left(\bar{U}_{i}\right)=0$. By (9), for any subgraph $H \subseteq F$ and $0 \leqslant i \leqslant n$, we have

$$
\begin{aligned}
& \sum_{v \in U_{i}}\left(\delta_{H}(v)-\varrho_{H}(v)\right)=\delta_{H}\left(U_{i}\right), \\
& \sum_{v \in \bar{U}_{i}}\left(\delta_{H}(v)-\varrho_{H}(v)\right)=-\varrho_{H}\left(\bar{U}_{i}\right) .
\end{aligned}
$$

Definition 4.7. For a splitted bipartite graph $G\left[A_{n}, B_{n}\right]$, let us define its flow representation $\vec{\nabla}(G)$ : take the symmetric difference $\nabla(G)=G\left[A_{n}, B_{n}\right] \triangle H_{0}(n)$, then orient the edges of $\nabla(G)$ such that each edge matches its orientation in $F_{n}$.

Lemma 4.8. The graph $\vec{\nabla}(G)$ is a flow realization of the degree sequence $\boldsymbol{d}$ of the splitted bipartite graph $G\left[A_{n}, B_{n}\right]$. Conversely, any flow realization of a splitted bipartite degree sequence $\boldsymbol{d}$ is the flow representation of some realization $G\left[A_{n}, B_{n}\right]$ of $\boldsymbol{d}$.

Proof. Observe the structure of $H_{0}(n)$ on Figure 2. We have

$$
\begin{aligned}
d_{H_{0}(n)}\left(a_{i}\right)-\operatorname{deg}_{G}\left(a_{i}\right) & =\operatorname{deg}_{\nabla(G)}\left(a_{i},\left\{b_{i}, \ldots, b_{n}\right\}\right)-\operatorname{deg}_{\nabla(G)}\left(a_{i},\left\{b_{1}, \ldots, b_{i-1}\right\}\right) \\
& =\delta_{\vec{\nabla}(G)}\left(a_{i}\right)-\varrho_{\vec{\nabla}(G)}\left(a_{i}\right), \\
\operatorname{deg}_{G}\left(b_{i}\right)-d_{H_{0}(n)}\left(b_{i}\right) & =\operatorname{deg}_{\nabla(G)}\left(b_{i},\left\{a_{i+1}, \ldots, a_{n}\right\}\right)-\operatorname{deg}_{\nabla(G)}\left(b_{i},\left\{a_{1}, \ldots, a_{i}\right\}\right) \\
& =\delta_{\vec{\nabla}(G)}\left(b_{i}\right)-\varrho_{\vec{\nabla}(G)}\left(b_{i}\right) .
\end{aligned}
$$

In the other direction, remove the orientation from the flow realization and take its symmetric difference with $H_{0}(n)$ to obtain an appropriate $G\left[A_{n}, B_{n}\right]$.

Corollary 4.9. For any splitted bipartite degree sequence $\boldsymbol{d} \in \mathbb{S}_{2 k}\left(\mathcal{H}_{0}\right)$ on $n+n$ vertices, the function $G \mapsto \vec{\nabla}(G)$ is a bijection between $\mathcal{G}(\boldsymbol{d})$ and flow realizations of $\boldsymbol{d}$ in $F_{n}$.

For example: every flow representation of a realization of

$$
\boldsymbol{h}_{0}(8)-\mathbb{1}_{a_{1}}+2 \cdot \mathbb{1}_{b_{2}}+\mathbb{1}_{a_{7}}-2 \cdot \mathbb{1}_{b_{8}}
$$

is a 3 -flow with sources at $a_{1}$ and $b_{2}$, and sinks as at $a_{7}$ and $b_{8}$; see Figure 3.

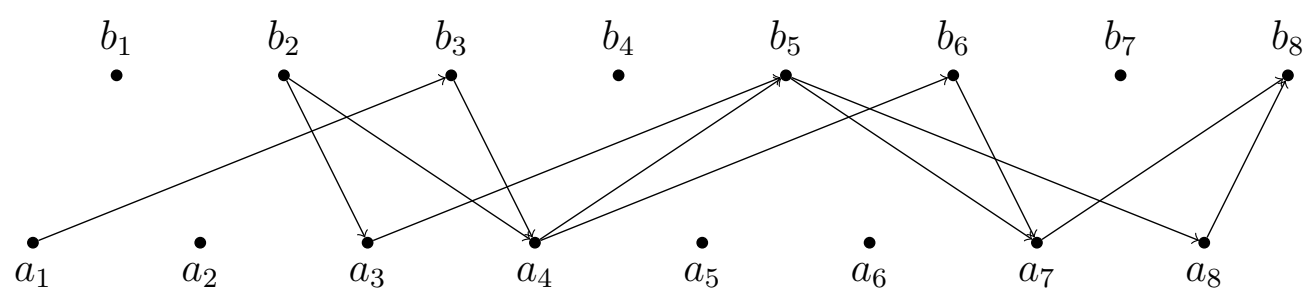

Figure 3: The flow representation of a realization of a degree sequence from $\mathbb{B}_{6}\left(h_{0}(8)\right)$. 


\section{Proof of Theorem 1.7: rapid mixing on $\mathbb{B}_{2 k}\left(\mathcal{H}_{0}\right)$}

\subsection{Overview of the proof}

Without loss of generality $\boldsymbol{d} \in \mathbb{S}_{2 k}\left(\mathcal{H}_{0}\right)$. Let $X, Y \in \mathcal{G}(\boldsymbol{d})$ be two distinct realizations. We will define a switch sequence

$$
\gamma_{X, Y}: X=Z_{0}, Z_{1}, \ldots, Z_{t}=Y
$$

We will also define a set of corresponding encodings

$$
L_{0}(X, Y), L_{1}(X, Y), \ldots, L_{t}(X, Y) \text {. }
$$

The canonical path system $\Gamma:=\left\{\gamma_{X, Y} \mid X, Y \in \mathcal{G}(\boldsymbol{d})\right\}$ on $G(\mathcal{M}(\boldsymbol{d}))$ will satisfy the following two properties:

- Reconstructible: there is an algorithm that for each $i$, takes $Z_{i}$ and $L_{i}(X, Y)$ as an input and outputs the realizations $X$ and $Y$.

- Encodable in $\mathcal{G}(\boldsymbol{d})$ : the total number of encodings on each vertex of $G(\mathcal{M}(\boldsymbol{d}))$ is at most a poly $_{k}(n)$ factor larger than $|\mathcal{G}(\boldsymbol{d})|$.

This proof technique was introduced by Jerrum and Sinclair [15] in the context of sampling matchings. Later, Kannan, Tetali, and Vempala [17] applied their technique to the switch chain.

The "Reconstructible" property ensures that the number of canonical paths traversing a vertex (and thus an edge) of the Markov graph $\mathcal{M}(\boldsymbol{d})$ is at most the size of the set of all possible encodings. Subsequently, by substituting into Equation (5), the "Encodable in $\mathcal{G}(\boldsymbol{d})$ " property implies that $\rho(\Gamma)=\mathcal{O}\left(\operatorname{poly}_{k}(n)\right)$. According to Lemma 3.5 , the last bound means that the bipartite switch Markov chain is rapidly mixing.

Now we give a description of how the $X=Z_{0}, Z_{1}, \ldots, Z_{t+1}=Y$ canonical path is constructed. The main idea is to morph $X$ into $Y$ "from left to right": a region of width proportional to $k$ called the buffer is moved peristaltically through $A_{n} \cup B_{n}$, consuming $X$ on its right and producing $Y$ on its left; see Figure 4 . In other words, the buffer is a sliding window which alternately extends and contracts.

The encoding $L_{i}(X, Y)$ will contain a realization whose structure is similar to $Z_{i}$, but the roles of $X$ and $Y$ are reversed; see Figure 4. Furthermore, $L_{i}(X, Y)$ will contain the position of the buffer and some additional information about the vertices in the buffer. 
The structure of a typical intermediate realization $Z_{i}$

\begin{tabular}{|c|c|c|c|c|}
\hline$b_{1} b$ & $b_{i}$ & \multirow{3}{*}{ Buffer } & $b_{i+z+1}$ & $b_{n-1} b_{n}$ \\
\hline \multicolumn{2}{|c|}{ Beginning of $Y$} & & \multicolumn{2}{|c|}{ End of $X$} \\
\hline$a_{1} a$ & $a_{i}$ & & $a_{i+z+1}$ & $a_{n-1} a_{n}$ \\
\hline
\end{tabular}

The realization in the corresponding encoding $L_{i}(X, Y)$

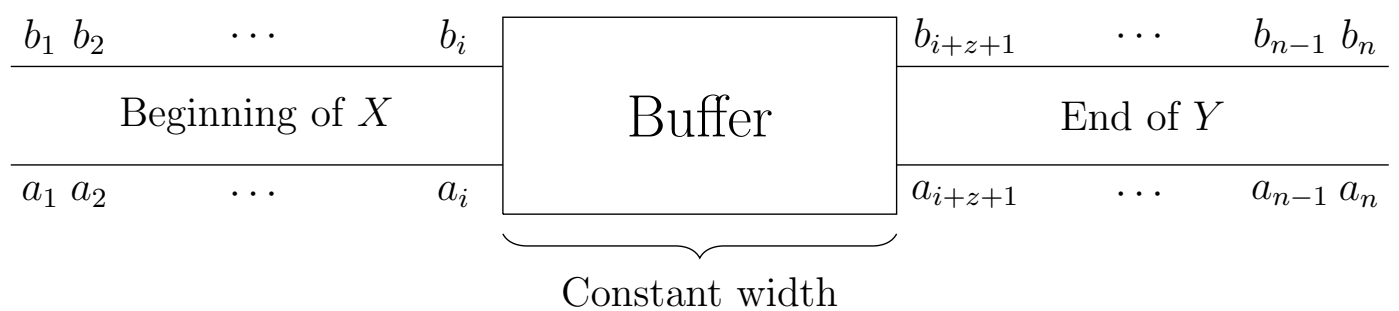

Figure 4: A (flow) realization along $\gamma_{X, Y}$ and the main part of the associated encoding. The width of the buffer is $z$. Edges (that are oriented left-to-right) are not shown to avoid clutter.

The following lemma shows the existence of a suitable buffer which can be used to interface two different realizations as displayed on Figure 4.

Lemma 5.1. Let $i, k, z \in \mathbb{N}$. Suppose that $z \geqslant 1$ if $k=1$, and $z \geqslant 2 k+\sqrt{2 k}+1$ if $k \geqslant 2$. If $0 \leqslant i \leqslant n-z$, then there is a realization $T_{X, Y}[i+1, i+z] \in \mathcal{G}(\boldsymbol{d})$ with buffer width $z$ and the following properties:

- $U_{i}$ induces identical subgraphs in $T_{X, Y}[i+1, i+z]$ and $Y$, and

- $\bar{U}_{i+z}$ induces identical subgraphs in $T_{X, Y}[i+1, i+z]$ and $X$.

Proof. We will work with flow representation in this proof. Since $X$ and $Y$ are the realizations of the same degree sequence, the source-sink distribution in their corresponding flow representation is identical. It is sufficient to design a flow $W$ which joins the flow $\vec{\nabla}(X)$ leaving $U_{i}$ and redirects it to the vertices in $\bar{U}_{i+z}$ with the same distribution as $\vec{\nabla}(Y)$ flows into them from $U_{i+z}$. The reason this is not trivial is because there are possibly sources and/or sinks in $U_{i+z} \backslash U_{i}$ which $W$ needs to account for.

The case $k=1$ can be manually checked at this point, since a 1 -flow is a directed path. In Figure 5 we show 4 different cases when the flow prescribed by $\boldsymbol{d}$ (Definition 4.6) has a source in $U_{i}$ and a sink in $\bar{U}_{i+1}$. If this is not the case, then $\vec{\nabla}(Y)$ or $\vec{\nabla}(X)$ is a good choice for $T_{X, Y}[i+1, i+z]$. 


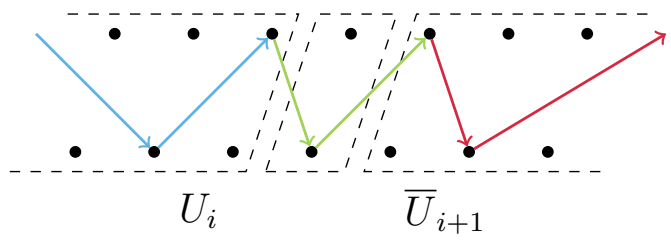

(a) The path $\vec{\nabla}(X)\left[U_{i}\right]$ ends with $b_{i}$ and $\vec{\nabla}(Y)\left[\bar{U}_{i+1}\right]$ starts with $b_{i+2}$.

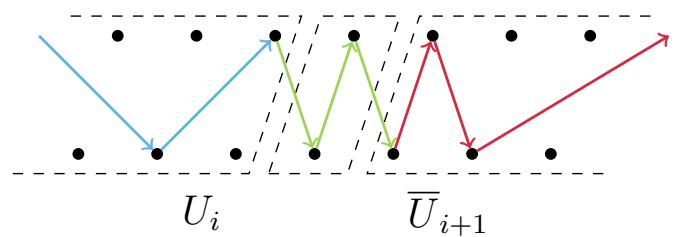

(c) The path $\vec{\nabla}(X)\left[U_{i}\right]$ ends with $b_{i}$ and $\vec{\nabla}(Y)\left[\bar{U}_{i+1}\right]$ starts with $a_{i+2}$.

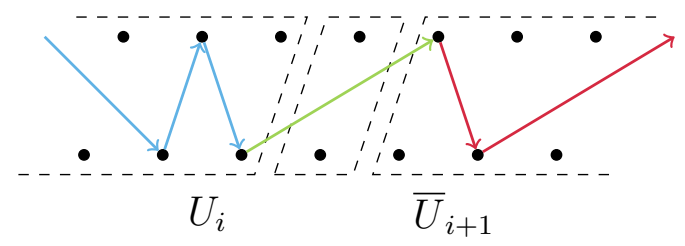

(b) The path $\vec{\nabla}(X)\left[U_{i}\right]$ ends with $a_{i}$ and $\vec{\nabla}(Y)\left[\bar{U}_{i+1}\right]$ starts with $b_{i+2}$.

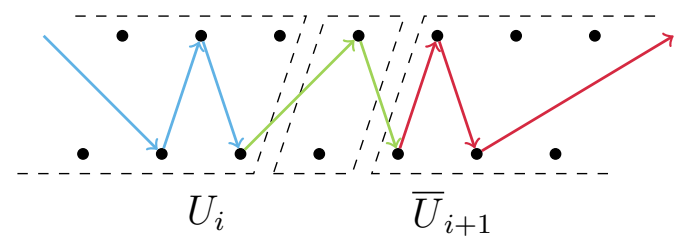

(d) The path $\vec{\nabla}(X)\left[U_{i}\right]$ ends with $a_{i}$ and $\vec{\nabla}(Y)\left[\bar{U}_{i+1}\right]$ starts with $a_{i+2}$.

Figure 5: Constructing the flow representation of $T_{X, Y}[i+1, i+z]$ for $k=z=1$. We differentiate between four cases based on which vertex class contains the last and first vertices of the paths $\vec{\nabla}(Y)\left[U_{i}\right]$ and $\vec{\nabla}(X)\left[\bar{U}_{i+1}\right]$, respectively. Blue arrows $\rightarrow: \vec{\nabla}(Y)\left[U_{i}\right]$; red arrows $\rightarrow: \vec{\nabla}(X)\left[\bar{U}_{i+1}\right]$; green arrows $\rightarrow$ : edges crossing the buffer or incident on a vertex of the buffer.

To achieve the outlined goal for any $k$, we define an auxiliary network $F^{\prime}$ and prescribe the flow corresponding to the buffer on it. Let $e_{D}(W, Z)$ be the number of edges of $D$ that are directed from $W$ to $Z$.

$$
\begin{aligned}
A_{Y} & :=\left\{a_{j} \in A_{i} \mid e_{\vec{\nabla}(Y)}\left(a_{j}, \bar{B}_{i}\right)>0\right\} \\
B_{Y} & :=\left\{b_{j} \in B_{i} \mid e_{\vec{\nabla}(Y)}\left(b_{j}, \bar{A}_{i}\right)>0\right\} \\
A_{X} & :=\left\{a_{j} \in \bar{A}_{i+z} \mid e_{\vec{\nabla}(X)}\left(B_{i+z}, a_{j}\right)>0\right\} \\
B_{X} & :=\left\{b_{j} \in \bar{B}_{i+z} \mid e_{\vec{\nabla}(X)}\left(A_{i+z}, b_{j}\right)>0\right\} \\
A^{\prime} & :=A_{Y} \cup\left(A_{i+z} \backslash A_{i}\right) \cup A_{X} \\
B^{\prime} & :=B_{Y} \cup\left(B_{i+z} \backslash B_{i}\right) \cup B_{X}
\end{aligned}
$$

The underlying network $F^{\prime}$ is a subgraph of $F$ :

$$
F^{\prime}:=F\left[A^{\prime}, B^{\prime}\right]-E\left(F\left[A_{Y} \cup A_{X}, B_{Y} \cup B_{X}\right]\right)
$$

i.e., the flow cannot use edges between $A_{X}, B_{X}, A_{Y}, B_{Y}$. Note, that to prove the lemma for $k=z=1$, one has to use edges of $F\left[A_{Y}, B_{X}\right]$ and $F\left[A_{X}, B_{Y}\right]$ in Figure $5(\mathrm{~b})$. For $k=1$ and $z \geqslant 2$, the edges of $F^{\prime}$ suffice to create a cross-over between the two flows. For the proof of the lemma for $k \geqslant 2$, we avoid using the edges of $F\left[A_{Y}, B_{X}\right]$ and $F\left[A_{X}, B_{Y}\right]$ to keep the analysis simple. 
The flow in the buffer will be a subgraph $W \subset F^{\prime}$. Let us define $f: A^{\prime} \cup B^{\prime} \rightarrow \mathbb{Z}$ :

$$
\begin{aligned}
f\left(a_{j}\right):= \begin{cases}e_{\vec{\nabla}(Y)}\left(a_{j}, \bar{B}_{i}\right), & \text { if } a_{j} \in A_{Y} \\
\operatorname{deg}_{H_{0}(n)}\left(a_{j}\right)-\operatorname{deg}_{\boldsymbol{d}}\left(a_{j}\right), & \text { if } a_{j} \in A_{i+z} \backslash A_{i} \\
-e_{\vec{\nabla}(X)}\left(B_{i+z}, a_{j}\right), & \text { if } a_{j} \in A_{X}\end{cases} \\
f\left(b_{j}\right):= \begin{cases}e_{\vec{\nabla}(Y)}\left(b_{j}, \bar{A}_{i}\right), & \text { if } b_{j} \in B_{Y} \\
\operatorname{deg}_{\boldsymbol{d}}\left(b_{j}\right)-\operatorname{deg}_{H_{0}(n)}\left(b_{j}\right), & \text { if } b_{j} \in B_{i+z} \backslash B_{i} \\
-e_{\vec{\nabla}(X)}\left(A_{i+z}, b_{j}\right), & \text { if } b_{j} \in B_{X}\end{cases}
\end{aligned}
$$

Let us prescribe the following equations on the difference between the in- and out-degrees of $W$ :

$$
\begin{aligned}
& \delta_{W}\left(a_{j}\right)-\varrho_{W}\left(a_{j}\right)=f\left(a_{j}\right) \quad \forall a_{j} \in A^{\prime}, \\
& \delta_{W}\left(b_{j}\right)-\varrho_{W}\left(a_{j}\right)=f\left(b_{j}\right) \quad \forall b_{j} \in B^{\prime} .
\end{aligned}
$$

If such a $W$ exists, then $\vec{\nabla}(Y)\left[A_{i}, B_{i}\right]+W+\vec{\nabla}(X)\left[\bar{A}_{i+z}, \bar{B}_{i+z}\right]$ is a flow representation of $\boldsymbol{d}$, which, according to Corollary 4.9, corresponds to a graph whose degree sequence is $\boldsymbol{d}$.

By Theorem 4.4, it is sufficient to show that for every $S \subseteq A^{\prime} \cup B^{\prime}$ we have

$$
\delta_{F^{\prime}}(S) \geqslant \sum_{v \in S} f(v)
$$

to conclude that a $W$ satisfying (15) exists. From now on, we focus on proving (16).

Recall (10). The right-hand side of (16) is at most $k$ :

$$
\begin{aligned}
\sum_{v \in S} f(v) & \leqslant \sum_{v \in A^{\prime} \cup B^{\prime}} f(v)^{+} \leqslant \sum_{v \in U_{i+z} \backslash U_{i}} f(v)^{+}+\sum_{a_{j} \in A_{Y}} e_{\vec{\nabla}(Y)}\left(a_{j}, \bar{B}_{i}\right)+\sum_{b_{j} \in B_{Y}} e_{\vec{\nabla}(Y)}\left(b_{j}, \bar{A}_{i}\right)= \\
& =\sum_{v \in U_{i+z} \backslash U_{i}} f(v)^{+}+\delta_{\vec{\nabla}(Y)}\left(U_{i}\right)=\sum_{v \in U_{i+z} \backslash U_{i}} f(v)^{+}+\sum_{v \in U_{i}} f(v) \leqslant k .
\end{aligned}
$$

It is sufficient to prove (16) for subsets $S$ for which $\delta_{F^{\prime}}(S)-\sum_{v \in S} f(v)$ is minimal. We claim that for every $S$ that satisfies the minimality condition, the following four statements hold:

- If $\left|S \cap\left(A_{i+z} \backslash A_{i}\right)\right|>k$, then $B_{X} \subset S$.

- If $\left|S \cap\left(B_{i+z} \backslash B_{i}\right)\right|>k$, then $A_{X} \subset S$.

- If $\left|S \cap\left(A_{i+z} \backslash A_{i}\right)\right|<z-k$, then $B_{Y} \cap S=\varnothing$.

- If $\left|S \cap\left(B_{i+z} \backslash B_{i}\right)\right|<z-k$, then $A_{Y} \cap S=\varnothing$.

We only prove the first statement because the rest can be shown by symmetry. Suppose $\left|S \cap\left(A_{i+z} \backslash A_{i}\right)\right|>k$ and $b_{j} \in B_{X}$, but $b_{j} \notin S$. Moving $b_{j}$ into $S$ changes the difference between the two sides of (16) by

$$
-\left|S \cap\left(A_{i+z} \backslash A_{i}\right)\right|-f\left(b_{j}\right)<-k+e_{\vec{\nabla}(X)}\left(A_{i+z}, b_{j}\right) \leqslant-k+\delta_{\vec{\nabla}(X)}\left(U_{i+z}\right) \leqslant 0,
$$


because $\vec{\nabla}(X)$ is an acyclic $k$-flow. Therefore we must have $B_{X} \subset S$.

Finally, we have four cases. In each case we show that (16) holds.

- Case 1: $\left|S \cap\left(A_{i+z} \backslash A_{i}\right)\right| \leqslant k$ and $\left|S \cap\left(B_{i+z} \backslash B_{i}\right)\right| \geqslant z-k$. We have

$$
\delta_{F^{\prime}}(S) \geqslant e_{F^{\prime}}\left(S \cap\left(B_{i+z} \backslash B_{i}\right),\left(A_{i+z} \backslash A_{i}\right) \backslash S\right) \geqslant \sum_{r=1}^{z-2 k-1} r \geqslant\left(\begin{array}{c}
z-2 k \\
2
\end{array}\right) \geqslant k,
$$

where the last inequality follows from $z \geqslant 2 k+\sqrt{2 k}+1$. Thus $S$ satisfies (16).

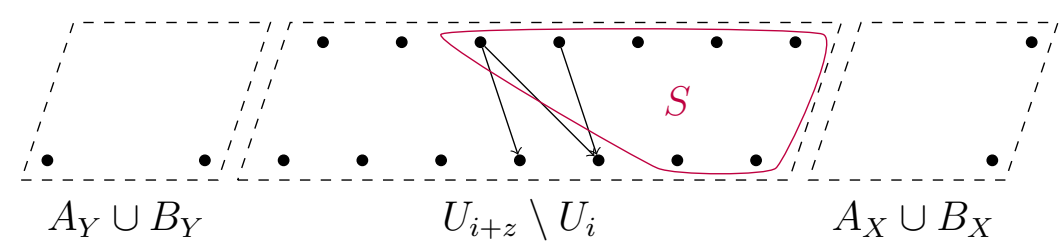

Figure 6: For the pictured selection of $S, e_{F^{\prime}}\left(S \cap\left(B_{i+z} \backslash B_{i}\right),\left(A_{i+z} \backslash A_{i}\right) \backslash S\right)$ is minimal if $S$ falls into Case $1, k=2$, and $z=7$. Only those edges are shown that leave $S$ and enter $U_{i+z} \backslash U_{i}$.

- Case 2: $\left|S \cap\left(A_{i+z} \backslash A_{i}\right)\right| \geqslant z-k$ and $\left|S \cap\left(B_{i+z} \backslash B_{i}\right)\right| \leqslant k$ : although this case is not completely symmetric to Case 1 , a similar proof shows that $\delta_{F^{\prime}}(S) \geqslant k$ (it is sufficient that $z \geqslant 2 k+\sqrt{2 k}$ ).

- Case 3: $\left|S \cap\left(A_{i+z} \backslash A_{i}\right)\right|>k$ and $\left|S \cap\left(B_{i+z} \backslash B_{i}\right)\right|>k$. By our previous statements, we have $A_{X} \cup B_{X} \subseteq S$. Consequently, the edges of $F$ leaving $S$ are either in $F^{\prime}$ or in $F\left[A_{Y}, B_{Y}\right]$. Therefore, using Equation (10), we have

$$
\begin{aligned}
\delta_{F^{\prime}}(S) & \geqslant \delta_{\vec{\nabla}(Y) \cap F^{\prime}}(S)=\delta_{\vec{\nabla}(Y)}\left(S \cup \bar{U}_{i+z}\right)-\delta_{\vec{\nabla}(Y) \cap F\left[A_{Y}, B_{Y}\right]}(S)= \\
& =\sum_{v \in S \cup \bar{U}_{i+z}}\left(\delta_{\vec{\nabla}(Y)}(v)-\varrho_{\vec{\nabla}(Y)}(v)\right)-\delta_{\vec{\nabla}(Y) \cap F\left[A_{Y}, B_{Y}\right]}(S)= \\
& =\sum_{v \in S \cap\left(U_{i+z} \backslash U_{i}\right)} f(v)-\varrho_{\vec{\nabla}(Y)}\left(\bar{U}_{i+z}\right)+\left(\delta_{\vec{\nabla}(Y)}\left(S \cap U_{i}\right)-\delta_{\vec{\nabla}(Y) \cap F\left[A_{Y}, B_{Y}\right]}(S)\right)= \\
& =\sum_{v \in S \cap\left(U_{i+z} \backslash U_{i}\right)} f(v)-\varrho_{\vec{\nabla}(X)}\left(\bar{U}_{i+z}\right)+\sum_{v \in S \cap U_{i}} e_{\vec{\nabla}(Y)}\left(v, \bar{U}_{i}\right)= \\
& =\sum_{v \in S \cap\left(U_{i+z} \backslash U_{i}\right)} f(v)-\sum_{v \in A_{X} \cup B_{X}} e_{\vec{\nabla}(X)}\left(U_{i+z}, v\right)+\sum_{v \in S \cap U_{i}} f(v)= \\
& =\sum_{v \in S \cap\left(U_{i+z} \backslash U_{i}\right)} f(v)+\sum_{v \in A_{X} \cup B_{X}} f(v)+\sum_{v \in S \cap U_{i}} f(v)=\sum_{v \in S} f(v),
\end{aligned}
$$

which is what we wanted to show. 
- Case 4: $\left|S \cap\left(A_{i+z} \backslash A_{i}\right)\right|<z-k$ and $\left|S \cap\left(B_{i+z} \backslash B_{i}\right)\right|<z-k$ : by our previous statements, we have $S \cap\left(A_{X} \cup B_{X}\right)=\varnothing$. Since $\delta_{F^{\prime}}(S)=\varrho_{F^{\prime}}\left(A^{\prime} \cup B^{\prime} \backslash S\right)$, the proof is practically the same as that of Case 3 , we can use $\vec{\nabla}(X)$ to demonstrate that (16) is satisfied by $S$.

\subsection{Constructing the canonical path $\gamma_{X, Y}$.}

We will explicitly construct $2(n-3 k-3)+1$ realizations along the switch sequence $\gamma_{X, Y}$. Let $X$ and $Y$ be the two different realizations which we intend to connect. The switch sequence includes $T_{X, Y}[i+1, i+3 k+1], T_{X, Y}[i+1, i+3 k+2], T_{X, Y}[i+2, i+3 k+2]$ for each $i=1, \ldots, n-3 k-3$ in increasing order. These realizations that we call milestones exist because of Lemma $5.1(z=3 k+1,3 k+2$ is sufficiently large). A roadmap is shown on Figure 7.

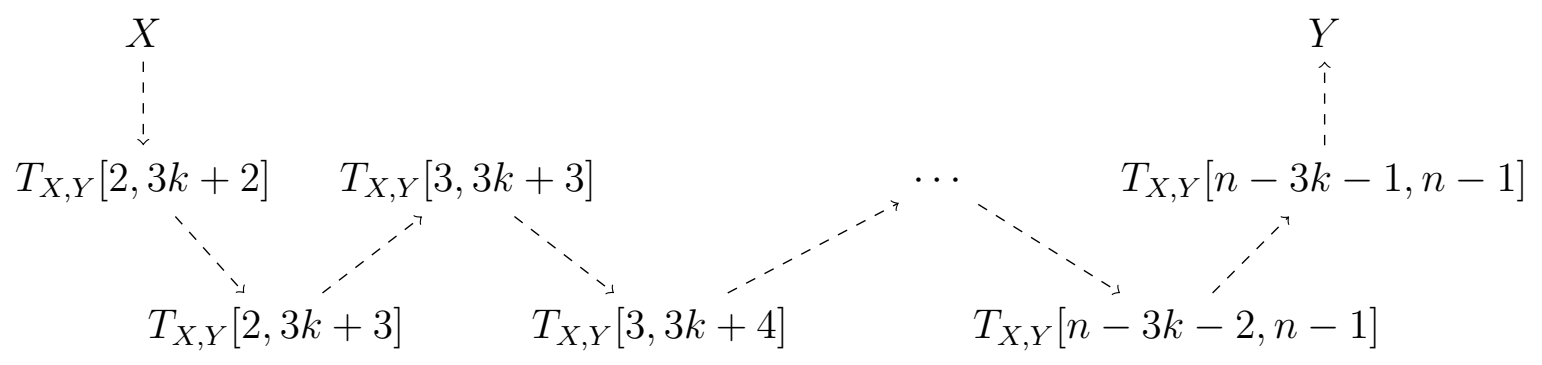

Figure 7: Roadmap of the switch sequence between $X$ and $Y$. Each dashed arrow $-\rightarrow$ represents a switch sequence of length $\mathcal{O}\left(k^{2}\right)$. The existence of a short switch sequence between milestones of the sequence is guaranteed by Lemma 5.2 .

Lemma 5.2. There is a switch sequence of length $\frac{1}{2}(5 k+2)^{2}$ that connects $T_{X, Y}[i+1, i+$ $3 k+1]$ to $T_{X, Y}[i+1, i+3 k+2]$ and $T_{X, Y}[i+1, i+3 k+2]$ to $T_{X, Y}[i+2, i+3 k+2]$.

Proof. The subgraphs of $\vec{\nabla}\left(T_{X, Y}[i+1, i+3 k+1]\right)$ and $\vec{\nabla}\left(T_{X, Y}[i+1, i+3 k+2]\right)$ induced by $U_{i} \cup \bar{U}_{i+3 k+2}$ are identical. By (10), the number of edges leaving $U_{i}$ and the number of edges entering $\bar{U}_{i+3 k+2}$ are both at most $k$ in a flow realization of $\boldsymbol{d}$. The set of source vertices of the at most $k$ edges leaving $U_{i}$ and the set of target vertices of the at most $k$ edges entering $\bar{U}_{i+3 k+2}$ are subsequently determined by the degree sequence $\boldsymbol{d}$ (see Definition 4.6), and are, therefore, identical too. The symmetric difference between $T_{X, Y}[i+1, i+3 k+1]$ and $T_{X, Y}[i+1, i+3 k+2]$ is restricted to edges induced by $U_{i+3 k+2} \backslash U_{i}$ and the at most $k+k$ source and target vertices of edges crossing this region. According to [5, Theorem 3.6], there is a switch sequence of length at most

$$
\frac{\left|E\left(T_{X, Y}[i+1, i+3 k+1]\right) \triangle E\left(T_{X, Y}[i+1, i+3 k+2]\right)\right|}{2} \leqslant \frac{1}{2}(5 k+2)^{2}
$$

between $T_{X, Y}[i+1, i+3 k+1]$ and $T_{X, Y}[i+1, i+3 k+2]$. This argument also holds for the switch distance between $T_{X, Y}[i+1, i+3 k+2]$ and $T_{X, Y}[i+2, i+3 k+2]$. 
Note that in Lemma 5.1, we may take $T_{X, Y}[1,3 k+2]:=X$ and $T_{X, Y}[n-3 k-1, n]:=Y$. By applying Lemma 5.2, the arrows in Figure 7 can be substituted with switch sequences of length $\frac{1}{2}(5 k+2)^{2}$. Concatenating these short switch sequences and pruning the circuits from the resulting trail (so that any realization is visited at most once by the canonical path) produces the switch sequence $\gamma_{X, Y}$ connecting $X$ to $Y$ in the Markov graph. The length of $\gamma_{X, Y}$ is at most

$$
\left|\gamma_{X, Y}\right| \leqslant \frac{1}{2}(5 k+2)^{2} \cdot n
$$

\subsection{Assigning the encodings.}

Each realization visited by $\gamma_{X, Y}$ receives an encoding that will be an ordered 4 -tuple consisting of another realization, two graphs of order $\mathcal{O}(k)$, and an integer in $\{1, \ldots, n\}$.

The neighborhood of a set of vertices $U \subseteq V(G)$ in a directed graph $G$ is denoted by

$$
N_{G}(U):=\{v \in V(G): \exists u \in U \text { such that } u v \in \vec{E}(G) \text { or } v u \in \vec{E}(G)\} .
$$

For the two graphs of order $\mathcal{O}(k)$ in the encoding we need the following definition.

Definition 5.3 (left-compressed neighborhood of the buffer). Let $H$ be a flow realization of $\boldsymbol{d}$, let $z \geqslant 1$ and $0 \leqslant i \leqslant n-z$. Let

$$
\begin{aligned}
R: & =\left\{j \in \mathbb{N}: 1 \leqslant j \leqslant i, e_{H}\left(a_{j}, \bar{U}_{i}\right) \neq 0 \text { or } e_{H}\left(b_{j}, \bar{U}_{i}\right) \neq 0\right\} \cup \\
& \cup\{j \in \mathbb{N}: i+1 \leqslant j \leqslant i+z\} \cup \\
& \cup\left\{j \in \mathbb{N}: i+z+1 \leqslant j \leqslant i, e_{H}\left(U_{i+z}, a_{j}\right) \neq 0 \text { or } e_{H}\left(U_{i+z}, b_{j}\right) \neq 0\right\} .
\end{aligned}
$$

In words, the elements of $R$ are subscripts of $a_{j}$ or $b_{j}$ that appear as a source or target of and edge leaving $U_{i}$ or entering $\bar{U}_{i+z}$. Let the elements of $R$ in increasing order be $\left(j_{t}\right)_{t=1}^{r}$ for some $j_{1}<\cdots<j_{r}$. Let $\sigma$ be a graph homomorphism which maps $a_{j_{t}} \mapsto a_{t}$ and $b_{j_{t}} \mapsto b_{t}$ for all $1 \leqslant t \leqslant r$ (edges are mapped vertex-wise). The left compressed copy of the closed neighborhood of the buffer $[i+1, i+z]$ in $H$ is

$$
\operatorname{cmpr}_{[i+1, i+z]}(H):=\sigma\left(H\left[\bigcup_{j \in R}\left\{a_{j}, b_{j}\right\}\right]\right) .
$$

To any realization on the canonical path $\gamma_{X, Y}$ we will assign an encoding

$$
L_{i}(X, Y):=\left(T_{Y, X}[i+1, i+3 k+2] ; \operatorname{cmpr}_{[i+1, i+3 k+2]}(\vec{\nabla}(X)) ; \operatorname{cmpr}_{[i+1, i+3 k+2]}(\vec{\nabla}(Y)) ; i\right)
$$

for some $0 \leqslant i \leqslant n-3 k-2$. Formally, each $L_{i}(X, Y)$ is an element of the Cartesianproduct of the set of realizations, a pair of left-compressed neighborhoods, and the set of non-negative integers. We will refer to the four elements as the object in the first, second, third, and fourth coordinates of the encoding. 


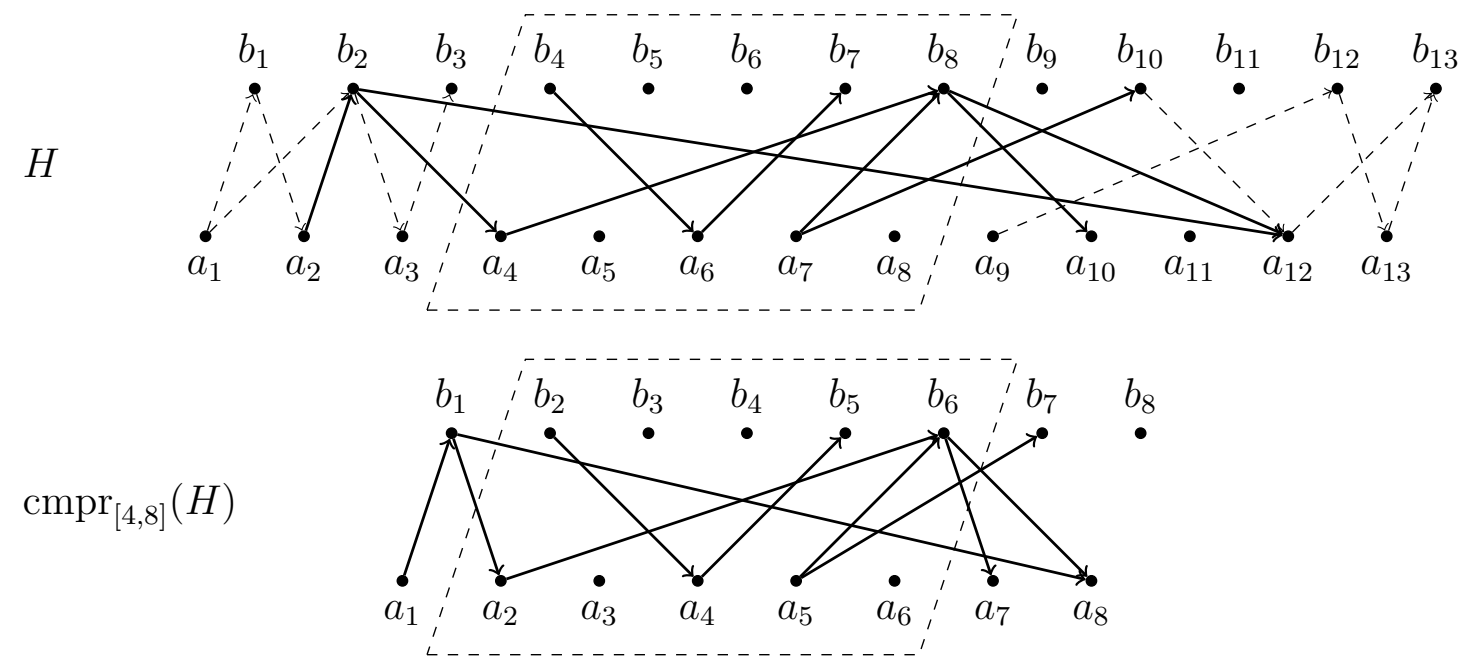

Figure 8: The flow $H$ shown in the upper half of the figure is shown left-compressed in the bottom half of the picture. The dashed $\rightarrow$ edges of $H$ are not included in its left-compressed image.

An encoding is assigned to each realization along the switch sequence $\gamma_{X, Y}$ as follows:

- The encoding $L_{0}(X, Y)$ (where $T_{Y, X}[1,3 k+2]:=Y$ ) is used from the beginning $X$ of the switch sequence until it arrives at $T_{X, Y}[2,3 k+2]$ (not including this realization).

- For $1 \leqslant i \leqslant n-3 k-3$, the encoding $L_{i}(X, Y)$ is used on the switch sequence from $T_{X, Y}[i+1, i+3 k+1]$ to $T_{X, Y}[i+1, i+3 k+2]$, and also from $T_{X, Y}[i+1, i+3 k+2]$ to $T_{X, Y}[i+2, i+3 k+2]$ (not included).

- The encoding $L_{n-3 k-2}(X, Y)$ (where $T_{Y, X}[n-3 k-1, n]:=X$ is chosen) is used on the switch sequence from $T_{X, Y}[n-3 k-1, n-1]$ to $Y$.

Since the number of vertices of $\operatorname{cmpr}_{[i+1, i+3 k+2]}(H)$ is at most $5 k+2$, the total number of possible encodings is at most

$$
\left|\left\{L_{i}(X, Y): X, Y \in \mathcal{G}(\boldsymbol{d}), 0 \leqslant i \leqslant n-3 k-2\right\}\right| \leqslant|\mathcal{G}(\boldsymbol{d})| \cdot 2^{2 \cdot(5 k+2)^{2}} \cdot n .
$$

\subsection{Estimating the load $\rho(\Gamma)$}

Lemma 5.4 (Reconstructability). Given $\boldsymbol{d}$, there is an algorithm that takes $Z_{i} \in \gamma_{X, Y}$ and $L_{i}(X, Y)$ as an input and outputs the realizations $X$ and $Y$ (for any $i$ ).

Proof. The first coordinate of $L_{i}(X, Y)$ is a realization, of the form $T_{Y, X}[i+1, i+3 k+2]$ for an unknown $X, Y$. The index $i$ is known, because it is the last component of $L_{i}(X, Y)$. By symmetry, it is sufficient to show how to recover $X$. By construction, $\vec{\nabla}(X)$ and $T_{Y, X}[i+1, i+3 k+2]$ induce identical graphs on $U_{i}$. Similarly, the induced subgraphs of 
$Z_{i}$ and $X$ on the vertices $\bar{U}_{i+3 k+2}$ are identical. Hence the only unknown part of $X$ is its induced subgraph on $\bigcup_{j \in R}\left\{a_{j}, b_{j}\right\}$, where

$$
R=\left\{j \in \mathbb{N}:\left\{a_{j}, b_{j}\right\} \cap N_{H}\left(U_{i+3 k+2} \backslash U_{i}\right) \neq \varnothing \text { or } i+1 \leqslant j \leqslant i+3 k+2\right\}
$$

as defined in (19). The subgraph in the second component of $L_{i}(X, Y)$ is the leftcompressed copy of $\vec{\nabla}(X)\left[\bigcup_{j \in R}\left\{a_{j}, b_{j}\right\}\right]$, so if we can "uncompress" it, we have the missing piece. Since edges of $\vec{\nabla}(X)$ do not enter $U_{i}$ from $\bar{U}_{i}$, their sources can be determined from the discrepancy between the degrees of $\vec{\nabla}(X)\left[U_{i}\right]$ and the differences prescribed in Definition 4.6:

$$
\begin{aligned}
& R_{A}^{\prime}:=\left\{j \in \mathbb{N}: 1 \leqslant j \leqslant i, \delta_{\vec{\nabla}(X)\left[U_{i}\right]}\left(a_{j}\right)-\varrho_{\vec{\nabla}(X)\left[U_{i}\right]}\left(a_{j}\right)<\operatorname{deg}_{H_{0}(n)}\left(a_{j}\right)-\operatorname{deg}_{\boldsymbol{d}}\left(a_{j}\right)\right\} \\
& R_{B}^{\prime}:=\left\{j \in \mathbb{N}: 1 \leqslant j \leqslant i, \delta_{\vec{\nabla}(X)\left[U_{i}\right]}\left(b_{j}\right)-\varrho_{\vec{\nabla}(X)\left[U_{i}\right]}\left(b_{j}\right)<\operatorname{deg}_{\boldsymbol{d}}\left(b_{j}\right)-\operatorname{deg}_{H_{0}(n)}\left(b_{j}\right)\right\}
\end{aligned}
$$

Similarly, the targets of the edges of $\vec{\nabla}(X)$ entering $\bar{U}_{3 k+2}$ are

$$
\begin{aligned}
& R_{A}^{\prime \prime}:=\left\{j \in \mathbb{N}: 1 \leqslant j \leqslant i, \delta_{\vec{\nabla}(X)\left[U_{i}\right]}\left(a_{j}\right)-\varrho_{\vec{\nabla}(X)\left[U_{i}\right]}\left(a_{j}\right)>\operatorname{deg}_{H_{0}(n)}\left(a_{j}\right)-\operatorname{deg}_{\boldsymbol{d}}\left(a_{j}\right)\right\} \\
& R_{B}^{\prime \prime}:=\left\{j \in \mathbb{N}: 1 \leqslant j \leqslant i, \delta_{\vec{\nabla}(X)\left[U_{i}\right]}\left(b_{j}\right)-\varrho_{\vec{\nabla}(X)\left[U_{i}\right]}\left(b_{j}\right)>\operatorname{deg}_{\boldsymbol{d}}\left(b_{j}\right)-\operatorname{deg}_{H_{0}(n)}\left(b_{j}\right)\right\}
\end{aligned}
$$

Observe, that $R=R_{A}^{\prime} \cup R_{B}^{\prime} \cup\{j \in \mathbb{N}: 1 \leqslant j \leqslant i\} \cup R_{A}^{\prime \prime} \cup R_{B}^{\prime \prime}$. Since left-compression preserves the order of the indices of $a_{j} \in A$ and $b_{j} \in B$, we are able to invert $\sigma$ (see Definition 5.3), and thus fully recover $\vec{\nabla}(X)$.

Proof of Theorem 1.7. By Lemma 5.4, for any given $Z \in \mathcal{G}(\boldsymbol{d})$ the number of pairs of realizations $X, Y \in \mathcal{G}(\boldsymbol{d})$ such that $Z \in \gamma_{X, Y}$ is bounded by the right hand side of (20). Substituting into (5),

$$
\rho(\Gamma) \leqslant 2^{2 \cdot(5 k+2)^{2}} \cdot n \cdot \max _{P(e) \neq 0} \frac{1}{P(e)} \leqslant 2^{2 \cdot(5 k+2)^{2}} \cdot n \cdot 6\left(\begin{array}{l}
n \\
4
\end{array}\right) \leqslant 2^{(5 k+2)^{2}} \cdot n^{5} .
$$

Let us apply Lemma 3.5. By $(18), \ell(\Gamma) \leqslant \frac{1}{2}(5 k+2)^{2} \cdot n$, thus $\tau(\varepsilon) \leqslant \mathcal{O}\left(n^{6} \cdot\left(n^{2}+\log \varepsilon^{-1}\right)\right)$, verifying that the switch Markov chain is rapidly mixing on $\mathbb{S}_{2 k}\left(\mathcal{H}_{0}\right)$ and $\mathbb{B}_{2 k}\left(\mathcal{H}_{0}\right)$.

\section{Proof of Theorem 1.9: non-stability of $\mathcal{H}_{k}$}

In this section we show that it is relatively straightforward to get the asymptotic growth rate of the number of realizations of $\boldsymbol{h}_{k}(n)$ when $k$ is a constant and $n$ tends to infinity. We first illustrate this for $k=1$. Recall Corollary 4.9 and that $\boldsymbol{h}_{1}(n)=\boldsymbol{h}_{0}(n)-\mathbb{1}_{a_{1}}-\mathbb{1}_{b_{n}}$.

Lemma 6.1. The number of all directed paths (integer 1-flows) from $a_{1}$ to $b_{n}$ in $F_{n}$ is

$$
\left[\begin{array}{l}
1 \\
1
\end{array}\right]^{T}\left[\begin{array}{ll}
2 & 1 \\
1 & 1
\end{array}\right]^{n-1}\left[\begin{array}{l}
0 \\
1
\end{array}\right] .
$$


Proof. Let $S_{1}(\ell)$ be the number of paths in $F_{n}$ that start at $a_{1}$ and end in $B_{\ell}$. Similarly, let $S_{2}(\ell)$ be the number of paths in $F_{n}$ that start at $a_{1}$ and end in one of the vertices in $A_{\ell}$. We have

$$
\begin{aligned}
& S_{1}(\ell+1)=2 S_{1}(\ell)+S_{2}(\ell), \\
& S_{2}(\ell+1)=S_{1}(\ell)+S_{2}(\ell) .
\end{aligned}
$$

Observe that $a_{1} \rightarrow b_{n}$ paths in $F_{n}$ are in bijection with paths starting at $a_{1}$ and ending in $A_{n}$ : the corresponding paths are obtained by deleting the last edge incident to $b_{n}$. Since $S_{1}(1)=S_{2}(1)=1$, from $(21)$ we get that $S_{2}(n)$ is the quantity in the statement of the Lemma and the proof is complete.

Corollary 6.2. As stated in (2), the number of realizations of $\boldsymbol{h}_{1}(n)$ is $\Theta\left(\left(\frac{3+\sqrt{5}}{2}\right)^{n}\right)$.

Proof. Neither $[1,1]$ nor $[0,1]^{T}$ is perpendicular to the eigenvector that belongs to the largest eigenvalue $\frac{3+\sqrt{5}}{2}$ of the matrix

$$
\left[\begin{array}{ll}
2 & 1 \\
1 & 1
\end{array}\right]
$$

The proof of Lemma 6.1 can be interpreted as follows. We count $a_{1} \rightarrow b_{n}$ paths by looking at their induced subgraphs on the vertices in $U_{\ell}$ (the number of these is precisely $S_{1}(\ell)+S_{2}(\ell)$ ). The main observation is that the number of ways an $a_{1} \rightarrow U_{\ell}$ path can be extended to an $a_{1} \rightarrow U_{\ell+1}$ path only depends on whether the path's endpoint lies in $A_{\ell}$ or in $B_{\ell}$.

Again, according to Corollary 4.9, realizations of $\boldsymbol{h}_{k}(n)$ are in a 1-to-1 correspondence with integer $k$-flows from $a_{1}$ to $b_{n}$. We shall mimic the argument of Lemma 6.1 with $k$-flows. The recursion will consider the beginning of a $k$-flow on $U_{\ell}$ and its "termination-type".

Definition 6.3 (set of types). Let $\mathcal{P}_{k}$ be the set of positive integer partitions of $k$ (the set of multisets of positive integers whose sum of elements is exactly $k$ ) and $\mathcal{P}_{0}:=\{\varnothing\}$. For all positive integers $k$, we define the set of types:

$$
\mathcal{T}_{k}:=\left\{(R, Q) \mid \exists 0 \leqslant m \leqslant k: R \in \mathcal{P}_{m}, Q \in \mathcal{P}_{k-m}\right\} .
$$

Definition 6.4 (type of a flow). Let $X$ be $k$-flow in $F_{n}\left[U_{\ell}\right]$ from a single source $a_{1}$, and the sinks are arbitrarily distributed in $U_{\ell}$. We say that the type of $X$ is $T=(R, Q) \in \mathcal{T}_{k}$ if there is an injective function $f: R \rightarrow A_{\ell}$ such that for every $a_{i} \in f(R)$ we have

$$
\varrho_{X}\left(a_{i}\right)-\delta_{X}\left(a_{i}\right)=f^{-1}\left(a_{i}\right),
$$

and for all $a_{i} \in A_{\ell} \backslash f(R)$ we have $\varrho_{X}\left(a_{i}\right)=\delta_{X}\left(a_{i}\right)$. Similarly, there is an injective function $g: Q \rightarrow B_{\ell}$ such that for every $b_{i} \in g(Q)$

$$
\varrho_{X}\left(b_{i}\right)-\delta_{X}\left(b_{i}\right)=g^{-1}\left(b_{i}\right),
$$

and for all $b_{i} \in B_{\ell} \backslash g(Q)$ we have $\varrho_{X}\left(b_{i}\right)=\delta_{X}\left(b_{i}\right)$. 
Informally, the type of $X$ describes the multiplicities of the incidences of the endpoints of the $k$-flow on $U_{\ell}$.

In the proof of Lemma 6.1 , the functions $S_{1}(\ell), S_{2}(\ell)$ were actually the number of 1 flows on $U_{\ell}$ of type $(\varnothing,\{1\})$ and $(\{1\}, \varnothing)$, respectively. The next definition is the analogue of the matrix in the proof of Corollary 6.2 for large $k$.

Definition 6.5 (type matrix). For all $k$, let us fix an ordering of the types: $\mathcal{T}_{k}=$ $\left(T_{1}, \ldots, T_{\left|\mathcal{T}_{k}\right|}\right)$. Let $\ell$ and $n$ be so large, that there exists a $k$-flow which has type $T_{i}$ on $U_{\ell}$. We define $p_{i, j}$ to be the number possible ways a $k$-flow on $U_{\ell}$ from the single source $a_{1}$ can be extended to a $k$-flow of type $T_{j}$ on $U_{\ell+1}$. We define the type-matrix $\mathcal{P}_{k}$ to be the $\left|\mathcal{T}_{k}\right| \times\left|\mathcal{T}_{k}\right|$ matrix whose element in the $i$-th row and $j$-th column is $p_{i, j}$.

It is not hard to see that $p_{i, j}$ is well-defined, in other words, $p_{i, j}$ does not depend on either $\ell, n$, or the $k$-flow.

In the proof of Corollary 6.2, the type matrix

$$
\mathcal{P}_{1}=\left[\begin{array}{ll}
2 & 1 \\
1 & 1
\end{array}\right]
$$

corresponds to the ordering $\mathcal{T}_{1}=((\varnothing,\{1\}),(\{1\}, \varnothing))$. Now we are ready to prove the analogue of Lemma 6.1 for $k$-flows where $k>1$.

Lemma 6.6. For every $k \geqslant 1$, the number of $k$-flows on $F_{n}$ from the single source $a_{1}$ to the single sink $b_{n}$ is

$$
v^{T} \mathcal{P}_{k}^{n-1} w
$$

where:

- $v$ is the vector of length $\left|\mathcal{T}_{k}\right|$ which contains 1 at the coordinates which correspond to the types $(\{k-1\},\{1\}),(\{k\}, \varnothing) \in \mathcal{T}_{k}$, and zero everywhere else,

- $\mathcal{P}_{k}$ is the type-matrix,

- $w$ is the vector of length $\left|\mathcal{T}_{k}\right|$ that contains 1 at the coordinate that corresponds to the type

$$
(\overbrace{\{1,1, \ldots, 1\}}^{k}, \varnothing) \in \mathcal{T}_{k}
$$

and zero everywhere else.

Proof. With the appropriate substitutions, the proof is identical to the proof of Lemma 6.1. The type of a $k$-flow on $U_{1}$ emanating from $a_{1}$ is either $(\{1\},\{k-1\})$ or $(\varnothing,\{k\})$. By the definition of $\mathcal{P}_{k}$, the vector $v^{T} \mathcal{P}^{n-1}$ contains the number of graphs on the vertices $U_{n}$ with a given type. Of these, the $k$-flows from $a_{1} \rightarrow b_{n}$ correspond to graphs with type $(\varnothing,\{1,1, \ldots, 1\})$ (deleting $b_{n}$ and the incident edges results in a $k$-flow of this type). Hence the statement of the lemma follows.

The following simple property of the type matrix will be used. 
Definition 6.7. A matrix $\mathcal{P}$ is primitive, if $\exists m$ for which every entry of $\mathcal{P}^{m}$ is positive.

Lemma 6.8. The type matrix $\mathcal{P}_{k}$ is primitive for any $k$.

Proof. For every type $t \in \mathcal{T}_{k}$ it is easy to design a $k$-flow $X$ such that the type of $X\left[U_{\ell}\right]$ (for some $\ell$ ) is $t$ and the type of $X\left[U_{\ell+k}\right]$ is $(\{1,1, \ldots, 1\}, \varnothing)$. Hence in $\mathcal{P}_{k}^{k}$ the row and column that correspond to the type $(\{1,1, \ldots, 1\}, \varnothing)$ are strictly positive. Since $\mathcal{P}_{k}$ is non-negative, it also follows that $\mathcal{P}_{k}^{2 k}$ is positive.

Now we are ready to prove the key lemma to refute the $P$-stability of the class of degree sequences $\mathcal{H}_{k}$.

Lemma 6.9. For every $k$, the largest eigenvalue of the type-matrix $\mathcal{P}_{k}$ is smaller than the largest eigenvalue of the type matrix $\mathcal{P}_{k+1}$.

Proof. By Lemma 6.8, both $\mathcal{P}_{k}$ and $\mathcal{P}_{k+1}$ are primitive. By the Perron-Frobenius theory, they both have a real positive eigenvalue $r_{k}$ and $r_{k+1}$, respectively, that is larger in absolute value than all of their other eigenvalues. Moreover, both limits

$$
\lim _{n \rightarrow \infty} \frac{\mathcal{P}_{k}^{n}}{r_{k}^{n}} \quad \text { and } \quad \lim _{n \rightarrow \infty} \frac{\mathcal{P}_{k+1}^{n}}{r_{k+1}^{n}}
$$

exist and are one dimensional projections. Let the set of types $S \subset \mathcal{T}_{k+1}$ be defined as follows:

$$
S:=\left\{(R, Q) \in \mathcal{T}_{k+1}: 1 \in R\right\} .
$$

Let $M^{(n)}$ be the principal minor of $\mathcal{P}_{k+1}^{n}$ that is obtained by taking those rows and columns which correspond to types in $S$. Without loss of generality, we may assume that if the $i$-th row of $M^{(1)}$ corresponds to a type $(R, Q)$, then the $i$-th row of $\mathcal{P}_{k}$ corresponds to the type $(R \backslash\{1\}, Q)$. Moreover, we may assume that the ordering of $\mathcal{T}_{k}$ and $\mathcal{T}_{k+1}$ is compatible in the following sense: if $T=\{R, Q\}$ and $T^{\prime}=\left(R^{\prime}, Q^{\prime}\right)$ are types in $S$ and $T<T^{\prime}$ according to the ordering on $\mathcal{T}_{k+1}$, then $(R \backslash\{1\}, Q)<\left(R^{\prime} \backslash\{1\}, Q^{\prime}\right)$ according to the ordering on $\mathcal{T}_{k}$.

First, we prove the following two properties of $M^{(1)}$.

1. The matrix $M^{(1)}$ is element-wise larger than or equal to $\mathcal{P}_{k}$.

2. The matrix $M^{(1)}$ is not equal to $\mathcal{P}_{k}$.

Since $|S|=\left|\mathcal{T}_{k}\right|$, the matrix $M^{(1)}$ is a $\left|\mathcal{T}_{k}\right| \times\left|\mathcal{T}_{k}\right|$ matrix. We start with proving the second statement. The entry of $\mathcal{P}_{k}$ in the intersection of the row and column that correspond to the type $(\{1, \ldots, 1\}, \varnothing) \in \mathcal{T}_{k}$ and $(\varnothing,\{k\}) \in \mathcal{T}_{k}$, respectively, is clearly 1 . On the other hand, the value of $M^{(1)}$ in this row and column corresponds to the number of transitions from $(\{1, \ldots, 1\}, \varnothing) \in \mathcal{T}_{k+1}$ to $(\{1\},\{k\})$ which is $k+1$ (the number of ways one can choose one of the $k+1$ paths which will not be extended). Therefore $M^{(1)} \neq \mathcal{P}_{k}$.

For the first statement, for any two types $(R, Q),\left(R^{\prime}, Q^{\prime}\right) \in \mathcal{T}_{k}$, if a type $(R, Q)$ subgraph of a $k$-flow on the vertices $U_{\ell}$ can be extended to an another type $\left(R^{\prime}, Q^{\prime}\right)$ subgraph on the vertices $U_{\ell+1}$ in $p$ ways, then clearly a type $(R \cup\{1\}, Q)$ subgraph of a $k+1$-flow on 
the vertices $U_{\ell}$ can be extended to a type $\left(R^{\prime} \cup\{1\}, Q^{\prime}\right)$ subgraph on the vertices $U_{\ell+1}$ in at least $p$ ways. Therefore the first property is also proven.

Suppose to the contrary that $r_{k+1} \leqslant r_{k}$. Since the limit

$$
\lim _{n \rightarrow \infty} \frac{\mathcal{P}_{k+1}^{n}}{r_{k+1}^{n}}
$$

exists and is finite, both the limits

$$
\lim _{n \rightarrow \infty} \frac{\mathcal{P}_{k+1}^{n}}{r_{k}^{n}} \text { and } \lim _{n \rightarrow \infty} \frac{M^{(n)}}{r_{k}^{n}}
$$

exist and are finite. Since $M^{(1)}$ is a principal minor of $\mathcal{P}_{k+1}$, and every element of $\mathcal{P}_{k+1}$ is non-negative, for all $k$ the matrix $M^{(k)}$ is element-wise larger than or equal to $\left(M^{(1)}\right)^{k}$. Hence the sequence

$$
\left\{\frac{\left(M^{(1)}\right)^{n}}{r_{k}^{n}}\right\}_{n=1}^{\infty}
$$

is bounded. By the two properties of $M^{(1)}$ and the fact that $\mathcal{P}_{k}$ is primitive, it follows that there is an integer $m$ such that $\left(M^{(1)}\right)^{m}$ is element-wise strictly larger than $\mathcal{P}_{k}^{m}$. Thus there is a positive $\varepsilon$ such that $\left(M^{(1)}\right)^{m}$ is element-wise strictly larger than $(1+\varepsilon) \mathcal{P}_{k}^{m}$. Therefore the sequence

$$
\left\{\frac{\left((1+\varepsilon) \mathcal{P}_{k}^{m}\right)^{n}}{r_{k}^{m n}}\right\}_{n=1}^{\infty}=\left\{(1+\varepsilon)^{n} \frac{\mathcal{P}_{k}^{m n}}{r_{k}^{m n}}\right\}_{n=1}^{\infty}
$$

is bounded, but this clearly contradicts the fact that the limit

$$
\lim _{n \rightarrow \infty} \frac{\mathcal{P}_{k+1}^{n}}{r_{k+1}^{n}}
$$

is a one dimensional projection.

Proof of Theorem 1.9. Observe, that $\left\|\boldsymbol{h}_{k+1}(n)-\boldsymbol{h}_{k}(n)\right\|_{1}=2$. However, according to Lemma 6.9

$$
\frac{\left|\mathcal{G}\left(\boldsymbol{h}_{k+1}(n)\right)\right|}{\left|\mathcal{G}\left(\boldsymbol{h}_{k}(n)\right)\right|}=\Theta\left(\left(\frac{r_{k+1}}{r_{k}}\right)^{n}\right)
$$

which grows exponentially as $n \rightarrow \infty$, so $\mathcal{H}_{k}$ is not $P$-stable.

\section{$7 \quad$ Strong stability and $H_{0}(\ell)$}

The degree sequence $\boldsymbol{h}_{0}(\ell)$ has other interesting properties. One is connected to the strong stability notion defined by Amanatidis and Kleer [1]. In their definition, they measure how stable a degree sequence is by measuring the maximum distance of a perturbed realization from the closest realization. 
Definition 7.1 (adapted from [1]). A degree sequence $\boldsymbol{d}$ is distance- $\ell$ strongly stable if for any realization $G^{\prime}$ of a degree sequence $d^{\prime}$ for which $\left\|d^{\prime}-d\right\|_{1} \leqslant 2$ there exists a realization $G$ such that $\left|E\left(G \triangle G^{\prime}\right)\right| \leqslant \ell$. A set of degree sequences is called strongly stable if there exists an $\ell$ such that every degree sequence in the set is distance- $\ell$ strongly stable.

The distance function $\left|E\left(G \triangle G^{\prime}\right)\right|$ used in Definition 7.1 differs from the function used in [1] up-to a factor of 2. Indeed, in one step, the Jerrum-Sinclair chain changes the size of the symmetric difference by at most 2 . In the other direction, suppose $G$ minimizes $\left|E\left(G \triangle G^{\prime}\right)\right|$. Take a vertex $v$ where $\boldsymbol{d}(v)=\boldsymbol{d}^{\prime}(v): E(G)$ and $E\left(G^{\prime}\right)$ evenly contribute to the edges incident to $v$ in $G \triangle G^{\prime}$. For the two vertices where $\boldsymbol{d}$ and $\boldsymbol{d}^{\prime}$ differ, there is an extra edge from $G$ or $G^{\prime}$. For this reason, if $G \triangle G^{\prime}$ is not a path, then it contains a cycle $C$ whose edges alternate between $G$ and $G^{\prime}$, hence $C$ is alternating (between edges and non-edges) in $G$ as well. However,

$$
\left|E\left((G \triangle C) \triangle G^{\prime}\right)\right|=\left|E\left(G \triangle G^{\prime}\right)\right|-|E(C)|,
$$

which contradicts the minimality of $G$. If $G \triangle G^{\prime}$ is path, the Jerrum-Sinclair chain needs at most $\left\lceil\frac{1}{2}\left|E\left(G \triangle G^{\prime}\right)\right|\right\rceil$ steps to transform $G^{\prime}$ into $G$.

The way we define strong stability immediately shows that strongly stable sets of degree sequences are also $P$-stable with $p(n)=n^{\ell+1}$.

Definition 7.2. We say that a bipartite graph $G[A, B]$ is covered by alternating cycles if for any $x \in A$ and $y \in B$ there exists a cycle $C$ which traverses (covers) $x y$ and alternates between the vertex sets $A$ and $B$, and also alternates between edges and non-edges of $G[A, B]$. Note the symmetry of the definition: a priori, we allow $x y \in E(G[A, B])$ and $x y \notin E(G[A, B])$ as well.

Lemma 7.3. The following statements are equivalent for a bipartite degree sequence $\boldsymbol{d}$.

(1) $\boldsymbol{d}$ is indecomposable;

(2) every $G \in \mathcal{G}(\boldsymbol{d})$ is covered by alternating cycles;

(3) every $\boldsymbol{d}^{\prime} \in \mathbb{S}_{2}(\boldsymbol{d})$ is graphic.

Proof. (1) $\Rightarrow(2)$ : Suppose $x y \in E(G)$ is not contained in an alternating cycle in $G$. Without loss of generality, we may suppose that $x \in A$. Let $A_{1} \subset A$ and $B_{1} \subset B$ be the set of vertices that are reachable from $x$ on an alternating path starting on a non-edge. Define $A_{2}=A \backslash A_{1}$ and $B_{2}=B \backslash B_{1}$. We must have $y \in B_{2}$, otherwise there is an alternating cycle on $x y$. Observe, that $G=G\left[A_{1}, B_{1}\right] \circ G\left[A_{2}, B_{2}\right]$, a contradiction. If $x y \notin E(G)$, the argument applies to the complements.

$(2) \Rightarrow(3)$ : Let $G \in \mathcal{G}(\boldsymbol{d})$ and $\boldsymbol{d}^{\prime} \in \mathbb{S}_{2}(\boldsymbol{d})$ be arbitrary. Suppose first, that $\boldsymbol{d}^{\prime}=$ $\boldsymbol{d}+\mathbb{1}_{x}+\mathbb{1}_{y}$ where $x \in A$ and $y \in B$. If $x y \notin E(G)$, then $G+x y$ is a realization of $\boldsymbol{d}^{\prime}$. If $x y \in E(G)$, take the alternating cycle $C$ on $x y$ in $G$, and observe that $G \triangle C+x y \in \mathcal{G}\left(\boldsymbol{d}^{\prime}\right)$.

If $\boldsymbol{d}^{\prime}=\boldsymbol{d}-\mathbb{1}_{x}-\mathbb{1}_{y}$ where $x \in A$ and $y \in B$, take the complement to arrive in the previous case. 
Finally, we have $\boldsymbol{d}^{\prime}=\boldsymbol{d}-\mathbb{1}_{x}+\mathbb{1}_{y}$ where $x, y \in A$ or $x, y \in B$. Without loss of generality, suppose that $x, y \in A$. Let $G \in \mathcal{G}(\boldsymbol{d})$ be arbitrary. If there is an alternating path $P$ starting on a edge from $x$ to $y$ in $G$, then $G \triangle P \in \mathcal{G}\left(\boldsymbol{d}^{\prime}\right)$. If there is no such alternating path, take a $z \in B$ such that $x z \in E(G)$. Then $y z \in E(G)$, too. As before, there exists an alternating cycle $C$ on $y z$ in $G$, because $\boldsymbol{d}$ is indecomposable. Since $C$ is an alternating cycle, $x z \notin E(G)$, thus $G \triangle C-x z+y z \in \mathcal{G}\left(\boldsymbol{d}^{\prime}\right)$.

$(3) \Rightarrow(1)$ : Suppose $\boldsymbol{d}$ is decomposable; let $G \in \mathcal{G}(\boldsymbol{d})$ and say $G=G_{1} \circ G_{2}$. Take $x \in V\left(G_{1}\right) \cap A$ and $y \in V\left(G_{2}\right) \cap B$ from distinct color classes, thus $x y \in E(G)$. If $\exists G^{\prime} \in \mathcal{G}\left(\boldsymbol{d}+\mathbb{1}_{x}+\mathbb{1}_{y}\right)$, then take $G \triangle G^{\prime}$ : there $x$ and $y$ have one extra edge in $G^{\prime}$ compared to $G$, therefore there is an alternating path joining $x$ to $y$ in $G$ starting on an non-edge, i.e., there is an alternating cycle on $x y$ in $G$. This means that there is a realization of $\boldsymbol{d}$ in which $x y$ is not an edge, which is a contradiction. Therefore $\boldsymbol{d}+\mathbb{1}_{x}+\mathbb{1}_{y}$ is not graphic. The proof is similar if $x \in V\left(G_{1}\right) \cap B$ and $y \in V\left(G_{2}\right) \cap A$ (take the complement).

By Theorem 2.5, to prove rapid mixing of the switch Markov chain on a set of degree sequences $\mathcal{D}$, it is sufficient to prove rapid mixing on indecomp $(\mathcal{D})$, defined in $(4)$. Lemma 7.3 implies that every element of $\mathbb{S}_{2}(\operatorname{indecomp}(\mathcal{D}))$ is graphic. As the next theorem shows, either every perturbation of an indecomposable degree is strongly distance- $(2 \ell)$ stable, or there is a realization of the indecomposable degree sequence which contains an induced copy of $H_{0}([\ell / 3\rceil)$.

Theorem 7.4. Suppose that in $G \in \mathcal{G}(\boldsymbol{d})$, the minimum length of an alternating cycle covering $x y$ is $2 \ell+2$. Then there is an induced copy of $H_{0}([\ell / 3\rceil)$ in $G$. If $d$ is indecomposable, then a graphic element of $\mathbb{S}_{2}(\boldsymbol{d})$ is not distance- $(2 \ell)$ strongly stable.

Proof. Notice that all of the conclusions are invariant on complementing $G$. If necessary, by taking the complement of $G$, we may suppose that $x y \notin E(G)$.

Let $C$ be an alternating cycle of length $2 \ell+2$ on $x y$. Let $a_{1}:=x$ and $b_{\ell+1}:=y$. Let $a_{i}$ and $b_{i}$ be the vertices at distance $2 i-2$ and $2 i-1$ from $x$ on $C-x y$, respectively.

Notice, that $a_{i} b_{j} \in E(G)$ if $i+1 \geqslant j$, and $a_{i} b_{j} \notin E(G)$ if $j \leqslant i-2$, otherwise $C$ is not the shortest alternating cycle on $x y$. Let

$$
\begin{aligned}
& A^{\prime}:=\left\{a_{3 i-2}: i=1, \ldots,\lceil\ell / 3\rceil\right\} \\
& B^{\prime}:=\left\{a_{3 i-1}: i=1, \ldots,\lceil\ell / 3\rceil\right\}
\end{aligned}
$$

We have

$$
G\left[A^{\prime}, B^{\prime}\right]=\left(a_{1}, \varnothing\right) \circ\left(\varnothing, b_{2}\right) \circ\left(a_{4}, \varnothing\right) \circ\left(\varnothing, b_{5}\right) \circ \cdots \circ\left(\varnothing, b_{3\lceil\ell / 3\rceil-1}\right) \simeq H_{0}(\lceil\ell / 3\rceil) .
$$

Take $\boldsymbol{d}^{\prime}:=\boldsymbol{d}+\mathbb{1}_{x}+\mathbb{1}_{y}$. For any realization $G^{\prime} \in \mathcal{G}\left(\boldsymbol{d}^{\prime}\right)$ we have $\left|E\left(G \triangle G^{\prime}\right)\right|>2 \ell$, otherwise there is an alternating path of length at most $2 \ell-1$ in $G$ which forms an alternating cycle of length $2 \ell$ with $x y$. Thus $\boldsymbol{d}^{\prime}$ is not distance- $(2 \ell)$ strongly stable. 


\section{Concluding remarks}

\subsection{Relationship to prior results}

Although the sets of degree sequences $\mathbb{B}_{2 k}\left(\mathcal{H}_{0}\right)$ (for some $k$ ) are certainly not diverse compared to the class of $P$-stable degree sequences, they are more numerous than, say, the regular degree sequences, for which rapid mixing of the switch Markov chain were first proven in $[2,19,13]$. Because $\mathbb{B}_{2 k}\left(\mathcal{H}_{0}\right)$ is not $P$-stable, the Jerrum-Sinclair chain [16] cannot produce a sample in polynomial expected time. Although in principle, the proof of rapid mixing on $P$-stable degree sequences [4] may be applicable to $\mathbb{B}_{2 k}\left(\mathcal{H}_{0}\right)$, we do not expect that it can be easily tweaked to accommodate it, for the following reasoning:

Let $\mathcal{T}$ be the set of $(X, Y)$ pairs of realizations of $\boldsymbol{h}_{1}(n)$ such that the paths $\vec{\nabla}(X)$ and $\vec{\nabla}(Y)$ are edge disjoint. It is simple to show that $|\mathcal{T}| \geqslant \exp (c n) \cdot\left|\mathcal{G}\left(\boldsymbol{h}_{1}(n)\right)\right|$, because for almost every realization $X$ we have $|E(\vec{\nabla}(X))| \approx \frac{2 n}{\sqrt{5}}$. For a pair $(X, Y) \in \mathcal{T}$, the edges $E(X) \triangle E(Y)$ form a cycle which traverses both $a_{1}$ and $b_{n}$. From this structure it follows that the multicommodity flow $\Gamma$ described in [4] between a pair of realizations $(X, Y) \in \mathcal{T}$ is a single switch sequence that passes through $H_{0}(n)-a_{1} b_{n} \in \mathcal{G}\left(\boldsymbol{h}_{1}(n)\right)$. Consequently, the load $\rho(\Gamma) \geqslant|\mathcal{T}| /\left|\mathcal{G}\left(\boldsymbol{h}_{1}(n)\right)\right| \geqslant \exp (c n)$ is exponential in $n$.

\subsection{Unconstrained graphs}

As mentioned in Section 2.1, $\Psi^{-1}$ embeds splitted bipartite graphs into the space of unconstrained graphs. The map $\Psi^{-1}$ preserves switches, since the symmetric difference of the edge sets of two realizations does not change by adding a clique to both graphs. Consequently, $\Psi^{-1}$ induces an isomorphism between the Markov-graphs $\mathcal{M}(\boldsymbol{d})$ and $\mathcal{M}(\boldsymbol{d})\left(\Psi^{-1}(\boldsymbol{d})\right)$.

Furthermore, through $\Psi^{-1}$, a set of canonical paths $\Gamma$ on $G(\mathcal{M}(\boldsymbol{d}))$ are mapped to a set of canonical paths $\Psi^{-1}(\Gamma)$ on $G\left(\mathcal{M}\left(\Psi^{-1}(\boldsymbol{d})\right)\right)$ satisfying

$$
\rho\left(\Psi^{-1}(\Gamma)\right) \leqslant \rho(\Gamma)
$$

In summary, Theorem 1.7 can be mapped unconstrained graphs: the switch Markov chain is rapidly mixing on $\Psi^{-1}\left(\mathbb{B}_{2 k}\left(\mathcal{H}_{0}\right)\right)$. Note, however, that

$$
\Psi^{-1}\left(\mathbb{B}_{2 k}\left(\mathcal{H}_{0}\right)\right) \subset \mathbb{B}_{2 k}\left(\Psi^{-1}\left(\mathcal{H}_{0}\right)\right),
$$

because the right hand side contains graphs that are not split.

\subsection{Possible generalizations}

The proof of Theorem 1.7 presented in Section 5 provides a polynomial bound on $\tau(\varepsilon)$ even for $k=\Theta(\sqrt{\log n})$, therefore:

Theorem 8.1. The switch Markov chain is rapidly mixing on

$$
\bigcup_{n=1}^{\infty} \mathbb{B}_{\sqrt{c \log n}}\left(\boldsymbol{h}_{0}(n)\right)
$$

for any constant $c>0$. 
We have not proved nor refuted $P$-stability of $\bigcup_{n=1}^{\infty} \mathbb{S}_{2 k}\left(\boldsymbol{h}_{0}(n)\right)$ when $k=\Theta(\sqrt{\log n})$.

We hope that the proof of Theorem 1.7 can be generalized to even broader degree sequence classes. By Theorem 4.4, an equivalent definition of $\boldsymbol{h}_{k}(n)$ is that for any realization $G \in \mathcal{G}\left(\boldsymbol{h}_{k}(n)\right)$, we have $\delta_{\vec{\nabla}(G)}\left(a_{1}\right)=k$, and for any $1 \leqslant i \leqslant n-1$ :

$$
\delta_{\vec{\nabla}(G)}\left(A_{i} \cup B_{i}\right)=\delta_{\vec{\nabla}(G)}\left(A_{i} \cup B_{i} \cup\left\{a_{i+1}\right\}\right)=k .
$$

Relax these constraints to requiring only that $\leqslant k$ edges leave $a_{1}, A_{i} \cup B_{i}$, and $A_{i} \cup B_{i} \backslash\left\{b_{i}\right\}$ each for every $i \in[1, n]$ : the set of graphs satisfying these is the set of realizations of a set of degree sequences we will call $\mathcal{H}_{\leqslant k}$. Naturally, $\mathbb{B}_{2 k}\left(\mathcal{H}_{0}\right) \subseteq \mathcal{H}_{\leqslant k}$, because a $k$-flow needs at most $k$ edges in any cut.

Conjecture 8.2. For any fixed $k$, the switch Markov chain is rapidly mixing on $\mathcal{H}_{\leqslant k}$.

We also put forward a conjecture inspired by the work Gao and Greenhill [11].

Definition 8.3 (Gao and Greenhill [11]). Let $\mathcal{D}$ be a set of graphic degree sequences and $k \in 2 \mathbb{N}$. We say that $\mathcal{D}$ is $\boldsymbol{k}$-stable, if there exists a polynomial $p \in \mathbb{R}[x]$ such that for any $n \in \mathbb{N}$ and any degree sequence $\boldsymbol{d} \in \mathcal{D}$ on $n$ vertices, any degree sequence $d^{\prime}$ with $\left\|d^{\prime}-d\right\|_{1} \leqslant k$ satisfies $\left|\mathcal{G}\left(\boldsymbol{d}^{\prime}\right)\right| \leqslant p(n) \cdot|\mathcal{G}(\boldsymbol{d})|$. The term $\boldsymbol{P}$-stable is an alias for 2-stable, which is the least restrictive non-trivial class defined here.

Gao and Greenhill [11] presented elegant conditions which when satisfied ensure 8stability of a class of degree sequences (8-stable degree sequence are by definition $P$-stable). In particular, they show that for $\gamma>2$, power-law distributed degree sequences are 8-stable, hence rapidly mixing. They also give a proof that 8-stable sets of degree sequence are rapidly mixing.

Conjecture 8.4. Suppose $\mathcal{D}$ is $(2 k+2)$-stable for some $k \in \mathbb{N}$. Then the switch Markov chain is rapidly mixing on $\mathbb{B}_{2 k}\left(\overline{\mathcal{D}^{\circ}}\right)$.

\section{References}

[1] Amanatidis, G., And Kleer, P. Rapid Mixing of the Switch Markov Chain for Strongly Stable Degree Sequences and 2-Class Joint Degree Matrices. In Proceedings of the Thirtieth Annual ACM-SIAM Symposium on Discrete Algorithms, Proceedings. Society for Industrial and Applied Mathematics, 2019, pp. 966-985.

[2] Cooper, C., Dyer, M., And Greenhill, C. Sampling Regular Graphs and a Peer-to-Peer Network. Combinatorics, Probability and Computing 16, 4 (2007), $557-593$.

[3] Durrett, R. Probability: Theory and Examples. Cambridge University Press, 2010.

[4] Erdős, P.L., Greenhill, C., Mezei, T. R., Miklós, I., Soltész, D., And Soukup, L. The mixing time of switch Markov chains: a unified approach. arXiv: 1903.06600 (2019). 
[5] Erdös, P.L., KirÁly, Z., And Miklós, I. On the Swap-Distances of Different Realizations of a Graphical Degree Sequence. Combinatorics, Probability and Computing 22, 3 (2013), 366-383.

[6] Erdős, P.L., Kiss, S. Z., Miklós, I., And Soukup, L. Approximate Counting of Graphical Realizations. PLOS ONE 10, 7 (2015), e0131300.

[7] Erdős, P.L., Mezei, T. R., Miklós, I., And Soltész, D. Efficiently sampling the realizations of bounded, irregular degree sequences of bipartite and directed graphs. PLOS ONE 13, 8 (2018), e0201995.

[8] Erdős, P.L., Miklós, I., And Toroczkai, Z. A Decomposition Based Proof for Fast Mixing of a Markov Chain over Balanced Realizations of a Joint Degree Matrix. SIAM Journal on Discrete Mathematics 29, 1 (2015), 481-499.

[9] Erdős, P.L., Miklós, I., And Toroczkai, Z. New Classes of Degree Sequences with Fast Mixing Swap Markov Chain Sampling. Combinatorics, Probability and Computing 27, 2 (2018), 186-207.

[10] Földes, S., And Hammer, P. L. Split graphs. In Proceedings of the Eighth Southeastern Conference on Combinatorics, Graph Theory and Computing (Louisiana State Univ., Baton Rouge, La., 1977) (1977), pp. 311-315. Congressus Numerantium, No. XIX.

[11] Gao, P., And Greenhill, C. Mixing time of the switch Markov chain and stable degree sequences. Discrete Applied Mathematics 291 (2021), 143-162.

[12] Greenhill, C., And Sfragara, M. The switch Markov chain for sampling irregular graphs and digraphs. Theoretical Computer Science 719 (2018), 1-20.

[13] Greenhill, C. S. A Polynomial Bound on the Mixing Time of a Markov Chain for Sampling Regular Directed Graphs. Electronic Journal of Combinatorics 18 \#P234 (2011).

[14] Hammer, P. L., And Simeone, B. The splittance of a graph. Combinatorica 1, 3 (1981), 275-284.

[15] Jerrum, M., McKay, B. D., And Sinclair, A. When is a Graphical Sequence Stable? University of Edinburgh, Department of Computer Science, 1989.

[16] Jerrum, M., And Sinclair, A. Fast uniform generation of regular graphs. Theoretical Computer Science 73, 1 (1990), 91-100.

[17] Kannan, R., Tetali, P., And Vempala, S. Simple Markov-chain algorithms for generating bipartite graphs and tournaments. Random Structures 86 Algorithms 14, 4 (1999), 293-308.

[18] Menger, K. Zur allgemeinen Kurventheorie. Fundamenta Mathematicae 10, 1 (1927), 96-115.

[19] Miklós, I., ERdős, P.L., And Soukup, L. Towards random uniform sampling of bipartite graphs with given degree sequence. Electronic Journal of Combinatorics 20, 1 (2013), \#P16. 
[20] Sinclair, A. Improved Bounds for Mixing Rates of Markov Chains and Multicommodity Flow. Combinatorics, Probability and Computing 1, 4 (1992), 351-370.

[21] Sinclair, A. Algorithms for Random Generation and Counting: A Markov Chain Approach. Progress in Theoretical Computer Science. Birkhäuser Basel, 1993.

[22] Tyshkevich, R. Decomposition of graphical sequences and unigraphs. Discrete Mathematics 220, 1 (2000), 201-238.

[23] Tyshkevich, R. I., And Chernyak, A. A. Decomposition of graphs. Cybernetics 21, 2 (1985), 231-242. 